\title{
Zebra finches go wild! Experimental cultural evolution of birdsong
}

\author{
Adriana Diez $^{\mathrm{a}, \mathrm{b}}$ and Scott A. MacDougall-Shackleton ${ }^{\mathrm{b}, \mathrm{c}, *}$ \\ ${ }^{a}$ Graduate Program in Neuroscience, University of Western Ontario, London, ON, Canada \\ ${ }^{\mathrm{b}}$ Advanced Facility for Avian Research, University of Western Ontario, London, ON, \\ Canada \\ ${ }^{c}$ Department of Psychology, University of Western Ontario, London, ON, Canada \\ *Corresponding author's e-mail address: smacdou2@uwo.ca
}

Received 3 July 2019; initial decision 24 September 2019; revised 1 December 2019; accepted 20 January 2020; published online 4 February 2020

\begin{abstract}
Vocal learning in songbirds is guided by experience and experience-independent factors. Previously, lineages of zebra finches founded by isolate-reared tutors showed cultural evolution to wild-type song. This suggests that experience-independent biases affect song development even in the absence of wild-type song. We hypothesized that cultural evolution of song depends on both experience-independent biases and tutor songs available. We predicted that songs more distant from wild-type would take longer to culturally evolve toward wild-type features. We bred zebra finches in three groups of lineages in which offspring of each generation served as tutors for the next. Lineages were founded with males singing wild-type song, isolate song, or heterospecific song. The two experimental lineages exhibited rapid cultural evolution of song with many temporal and spectral features converging to wild-type within two generations. However the rate of change differed depending on song features measured, and took longer for lineages founded with heterospecific song.
\end{abstract}

\section{Keywords}

birdsong, song learning, zebra finch, Bengaleses finch, cultural evolution.

\section{Introduction}

Birdsong is one of the most extensively studied forms of animal communication and has become an important model to study imitative vocal learning. Unlike language, imitative vocal learning is not unique to humans and is 
widespread among birds (Jarvis, 2007; Bolhuis et al., 2010). Birdsong learning and speech development share many features including progressive development of vocalizations, the influence of social interactions, and a critical role of auditory feedback (Doupe \& Kuhl, 1999). Songbirds have, therefore, become a common behavioral and neural model to study vocal learning as a perceptual and motor ability (Brainard \& Doupe, 2002).

Birdsong development is guided by multiple experiential factors including exposure to tutor songs and social interactions (Baptista \& Petrinovich, 1986; Houx \& Ten Cate, 1998; Beecher \& Brenowitz, 2005; Soma, 2011; Ljubicic et al., 2016; Beecher, 2017). Songbirds raised in captivity will typically learn the song of conspecifics to which they are exposed. In some cases birds will even learn the songs of heterospecifc tutors (Immelmann, 1972; Baptista \& Morton, 1981; Baptista \& Petrinovich, 1984; Clayton, 1989). Additionally, social feedback from females shapes the development of male song in cowbirds (Molothrus ater) and zebra finches (Taeniopygia guttata) (e.g., King \& West, 1983; West \& King, 1988; Carouso-Peck \& Goldstein, 2019). The effects of social experience on song development extend beyond interactions with female receivers, and include social interactions amongst young peers during the song learning process (Chaiken et al., 1997; Poirier et al., 2004).

In addition to social experience, the experience of hearing one's own vocalizations -auditory feedback- is critical to song development. For example, seminal experiments on deafened white-crowned sparrows (Zonotrichia leucophys nuttalli) and Mexican juncos (Junco phaeonotus) showed that the songs produced by deafened birds lacked both fundamental species-specific and dialectal features (Konishi, 1964, 1965). The fact that songs of deafened birds have fewer species-typical features indicates the importance of auditory feedback. In addition to experience with tutor songs and auditory feedback, developmental experience in general can affect song learning (Spencer \& MacDougall-Shackleton, 2011; MacDougall-Shackleton \& Spencer, 2012; Peters et al., 2014).

Beyond the critical role of experience in birdsong development, experience-independent factors also guide song development in many species. Shortly after the discovery of the importance of experience with a tutor song for song learning, it also became clear that songbirds have predispositions to learn species-typical sounds. Young swamp sparrows (Melospiza georgiana) learned only conspecific syllables when tutored with both swamp 
sparrow and song sparrow (M. melodia) syllables (Marler \& Peters, 1977). Furthermore, the songs of isolate-reared birds contain many species-typical features, indicating experience-independent predisposition to produce certain vocalizations (Thorpe, 1954; Konishi, 1965; Price, 1979; Williams et al., 1993; Lachlan \& Slater, 1999). Despite these experience-independent biases, many species of songbird will indeed learn to imitate heterospecific sounds if conspecific tutors are not available (e.g., Baptista \& Petrinovich, 1984), and some species routinely learn heterospecific song through vocal mimicry (Dalziell et al., 2014). Thus, song development depends on an interaction between social and auditory experience and experience-independent biases.

Birdsong development is thus a clear example of gene-culture interaction (Lachlan \& Slater, 1999). Imitative vocal learning is produced by an interaction between genes that bias the recognition and learning of conspecific song features, as well as the culturally transmitted songs themselves and social interactions (Lachlan \& Slater, 1999; Lachlan \& Feldman, 2003; Lachlan et al., 2004; Whiten et al., 2017). Social interactions can augment experienceindependent biases in song development (e.g., Volman \& Khanna, 1995; Smith et al., 2000). For example, pair-housed starlings (Sturnus vulgaris) with no adult tutors developed songs with more species-typical features than individually isolated starlings (Chaiken et al., 1997). However, experienceindependent biases affect which song features are learned from a social tutor. Wild-type zebra finch (Taeniopygia guttata) song features emerged de novo within a few generations in lineages of finches founded by birds singing isolate songs (Fehér et al., 2009). Young zebra finches exposed to this abnormal song, through immitation and accumulation of changes over generations, modified the original isolate song towards a song with wild-type features, showing learning biased towards wild-type zebra finch features. However, in the Fehér et al. (2009) study it was not determined if the rapidity of the cultural evolution over generations depended on how similar the initial tutor song was to the tutees' experience-independent biases.

In this study our objective was to explore the interaction of (i) experienceindependent biases in song learning and (ii) the nature of the tutor song in the cultural evolution of phonological features of song across generations. Building on the study by Fehér et al. (2009), we hypothesized that cultural evolution towards species-typical spectral and temporal song features should be more rapid if the song of the founding generation has more species-typical features. To test this, we created three groups of social lineages founded 
by song tutors singing wild-type zebra finch song (control group), isolate song, or heterospecific song. We predicted that, over generations, the isolatetutored song lineages should more rapidly evolve toward wild-type song features than the heterospecific-tutored lineages, as the latter contains fewer species-typical song features.

\section{Materials and methods}

\subsection{Study subjects and general procedures}

All the experimental procedures, care, and housing conditions followed the guidelines of the Canadian Council on Animal Care and were approved by the University of Western Ontario's Animal Use Subcommittee.

Male zebra finches used in this experiment were bred from a colony maintained at the Advanced Facility for Avian Research (AFAR) at the University of Western Ontario. We paired males and females in individual breeding cages with a nest cup and ad libitum access to multi-vitamin seeds, cuttlefish bone, grit, and water. During breeding, we provided one tablespoon (approx. $15 \mathrm{~g}$ ) of egg-food mix (blended hard-boiled egg and bread) daily. Once the chicks hatched we increased the amount of egg food mix to one tablespoon per chick until age of $45 \pm 2$ days post hatch (dph). During the entire experiment, the rooms were kept at $24^{\circ} \mathrm{C}$ and on a $14 \mathrm{~h}: 10 \mathrm{~h}$ light:dark photoperiod. The breeding process took over a year and a half, and was coordinated with the song tutoring process.

Both parents reared the young until $10 \mathrm{dph}$ of the oldest chick in the clutch. On that day, we moved the offspring and their mothers to a nursery room with other females (who do not sing) and chicks to avoid the offspring being exposed to their father's or other male's song, until $35 \pm 2 \mathrm{dph}$, when the young zebra finches reached feeding independence. Consequently, our protocol should have limited males from hearing or learning the songs of non-target males (Roper \& Zann, 2006). Offspring were genetically sexed at about $20 \mathrm{dph}$, via collection of a small blood sample, DNA extraction and PCR amplification of sex chromosome markers.

Once offspring reached feeding independence (approx. $35 \mathrm{dph}$ ) the male offspring (pupils) were randomly assigned to one of the three song tutoring conditions or lineages (Figure 1). In general, our tutoring protocol was similar to that of Fehér et al. (2009). In the tutoring conditions, each pupil was individually housed with a mature $(+120 \mathrm{dph})$, unrelated zebra finch 


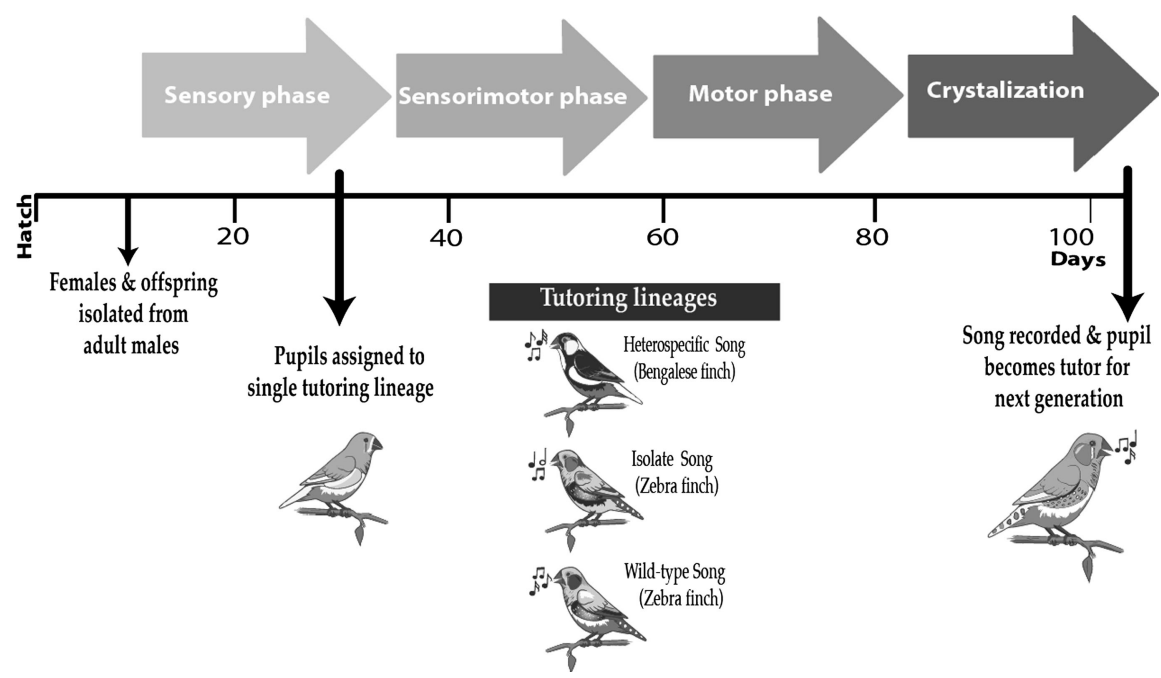

Figure 1. General timeline and experimental procedure. Young male zebra finches were isolated from their father, then assigned to one of three types of tutoring lineages (conspecific, heterospecific, or isolate song). For each generation, pupils from the prior generation were used as tutors for the next, to create lineages over which cultural evolution through song learning could occur.

male (from now on the tutor) for 90 days. At this point the pupil's song was recorded twice, with 7 days in between, to confirm that the song was crystalized (confirmed in all cases). After their songs were recorded, the pupils were assigned as tutors for a new pupil generation. This process was repeated for 4 generations in total.

We used the above procedures to create three types of song tutoring lineages. Similar to a previous study we created isolate song tutoring lineages founded by birds singing isolate song (Fehér et al., 2009). In addition, we created two more types of lineages: control lineages founded by zebra finches that sang wild-type song, and heterospecific tutored lineages of zebra finches that were initially tutored by Bengalese finches (Lonchura striata domestica). In total we produced 79 male zebra finches from 26 clutches produced by 40 breeding pairs of finches. The final sample size of males recorded in each group and generation are indicated in Table 1. In both the isolate song and wild type song lineages we reused a single tutor for two successive pupils at different times. This resulted in sample sizes increasing by one over generations (Table 1). In the heterospecific lineages the first generation was group tutored, resulting in a larger number of birds in generation 1 than there were 
Table 1.

Number of males per generation for each lineage.

\begin{tabular}{lccc}
\hline & $\begin{array}{c}\text { Heterospecific Song } \\
\text { (Bengalese Finch) }\end{array}$ & $\begin{array}{c}\text { Isolate Song } \\
\text { (Zebra finch) }\end{array}$ & $\begin{array}{c}\text { Wild Type Song } \\
\text { (Zebra finch) }\end{array}$ \\
\hline Tutor Generation & 6 & 7 & 7 \\
Generation 1 & 13 & 8 & 8 \\
Generation 2 & 14 & 8 & 6 \\
Generation 3 & 7 & 3 & 3 \\
Generation 4 & 5 & 2 & 2 \\
\hline
\end{tabular}

The tutor generation of the heterospecific song lineage were Bengalese finches. All other birds were zebra finches.

tutors. In all lineages we eventually had reduced breeding success, which resulted in reductions in sample sizes over generations (Table 1).

\subsection{Tutoring lineages}

\subsubsection{Heterospecific song lineages}

The first generation of subjects in the heterospecific song condition were exposed to heterospecific songs from six adult male Bengalese finches. These six males were housed as three same sex pairs that received zebra finch eggs from the colony that they incubated, hatched and raised in groups, producing 13 foster sons for the first experimental generation. In subsequent generations we housed one tutor and one pupil at a time together in individual cages.

We chose Bengalese finches as heterospecific tutors because male zebra finches tutored by Bengalese finches are able to imitate Bengalese finch song (Clayton, 1987). Bengalese finch syllable types and syllable syntax rarely appear in wild-type zebra finch songs (Funabiki \& Konishi, 2003), but zebra finches reared by Bengalese finch do learn some components of Bengalese finch song (Clayton, 1987). An example of Bengalese finch song is illustrated in Figure 2.

\subsubsection{Isolate song lineages}

The first generation of subjects in the isolate song condition were exposed to isolate zebra finch songs. To obtain the baseline tutor group we used seven male zebra finches from the zebra finch colony. The breeding conditions to produce these males were the same as described above, however, when the young males reached independence at age $35( \pm 2)$ dph, they were individually housed in a soundproof attenuation chamber until age $140 \mathrm{dph}$. 

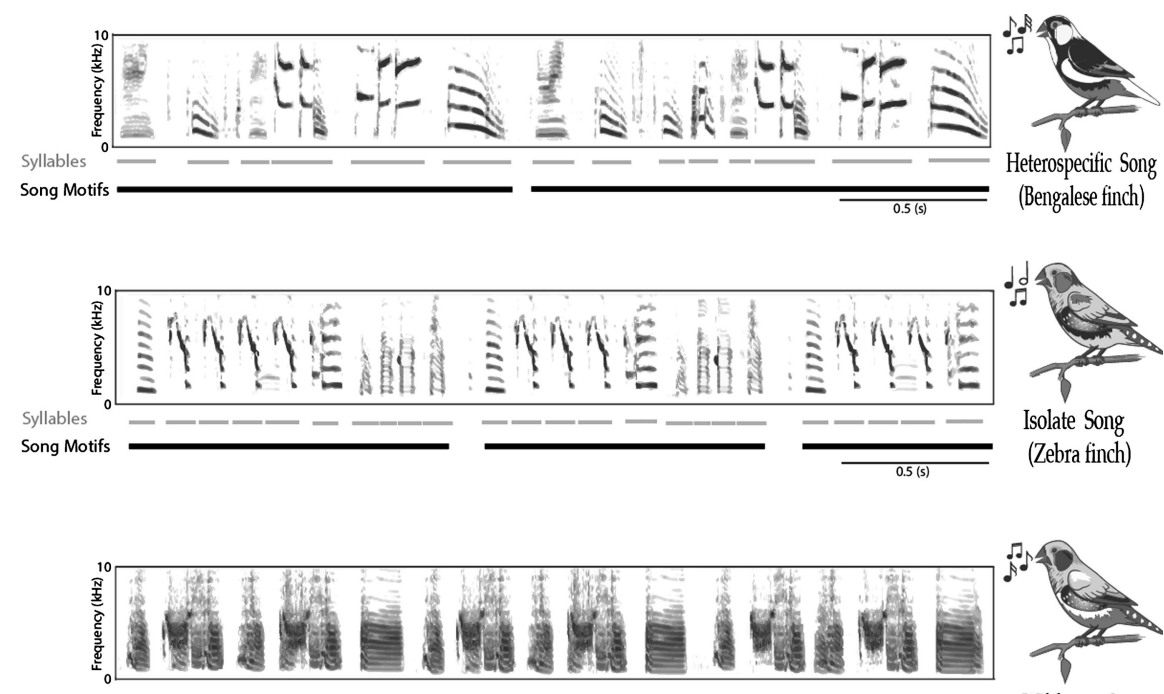

Syllables
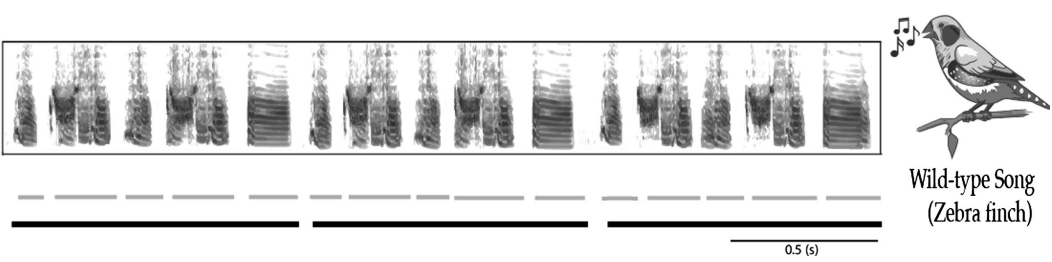

Figure 2. Example sound spectrograms of heterospecific, isolate and wild-type songs from the initial tutor generation. Song bouts, motifs, and syllables are indicated by horizontal lines.

An isolate song develops when males are kept in such social and acoustic isolation throughout their song-learning period (day 10-90; Williams et al., 1993). An example of the isolate song produced by these isolte song tutors is illustrated in Figure 2.

\subsubsection{Wild-type conspecific song lineages}

The first generation of subjects in the wild-type conspecific condition were exposed to wild-type zebra finch song from a baseline (tutor group) over three generations. To obtain the baseline tutor group we used seven adult male zebra finches from our zebra finch breeding colony that all sang a wildtype song (Figure 2).

\subsection{Song recording}

We recorded female-directed songs from all the tutors and pupils from the three tutoring groups described above (heterospecific song, isolate song, wild-type song). The songs were recorded after song crystallization at the age of $120 \mathrm{dph}$ or older. To obtain the song recording, each male was kept in isolation in a sound attenuation chamber for around $20 \mathrm{~h}$. Following this isolation period, a conspecific female was placed in an adjacent cage in the 
chamber to motivate the male to sing. These directed songs were obtained from two recording sessions of $10 \mathrm{~min}$. We had an interval of seven days between each session to guarantee song crystallization (which occurred in all cases). Songs were digitally recorded using an omni-directional microphone (Sennheiser ME62/K6P) and a digital audio recorder (Marantz PMD671) with a sampling rate of $44.1 \mathrm{kHz}$ and 32-bit per second.

\subsection{Song analysis}

Zebra finch and Bengalese finch songs are produced in bouts of uninterrupted singing. These bouts consist of song motifs, a consistent repetition of at least two syllables. Syllables are composed of one or more highly structured vocal elements (Figure 2). For this analysis, we visually inspected the spectrograms using Raven Pro Software (version 1.4, Cornell Lab of Ornithology, Ithaka, NY, USA), and we choose the longest song bout produced by the subject in its first recording session. Then, from the selected song bout we choose the song motif that had the most recurrent and complete syllable sequence (Holveck et al., 2008), as well as a silence interval $>500 \mathrm{~ms}$ (clearly distinguishable from syllable gaps) between each motif. Syllable segmentation was based on the stereotyped cluster of element sequences and silence intervals $<500 \mathrm{~ms}$ (James \& Sakata, 2019) as well as automatic segmentation by Sound Analysis Pro 2011 (version 2011.107; freely available at: http://soundanalysispro.com, for more information see Tchernichovski et al., 2000).

The segmentation process allowed us to analyze the songs at three levels: song-bouts, song-motifs and syllables, for further comparisons between lineages and generations (Figure 2). Introductory syllables were not included in the measurements. Our motif analyses included repeated syllables, and our bout analysis included repeated motifs, to characterize temporal (Table 2) and spectral (Table 3) features at each level of organization. We did not attempt to identify individual syllables and characterize copying accuracy. To obtain all the quantitative measurements for the three levels (song-bouts, song-motifs and syllables) we used Sound Analysis Pro (SAP) 2011 (version 2011.107; Tchernichovski et al., 2000) SAP automatically extracted measurements of mean, minima, maxima and variances for 9 different traits: amplitude, pitch, mean frequency, goodness of pitch, AM, FM, Wiener entropy, continuity over time (t), continuity over frequency (f) (Table 2). We included the measurement of mean, minima and maxima values for every 
Table 2.

Temporal features of song bouts, song motifs and song syllables measured using Sound Analysis Pro (version 2011.107; Tchernichovski et al., 2000).

\begin{tabular}{|c|c|c|}
\hline Feature & Feature description & $\begin{array}{l}\text { Linear } \\
\text { Discriminant } \\
\text { Function } \\
\text { analyses }\end{array}$ \\
\hline \multicolumn{3}{|l|}{ Song bout features } \\
\hline Duration & $\begin{array}{l}\text { Total duration of song bouts, including silent gaps } \\
\text { between motifs }\end{array}$ & \\
\hline Song motif rate & Number of song motifs per song bout & bout ldf \\
\hline Song motif density & Number of song motifs produced per second & bout ldf \\
\hline Song motif distance & Mean duration of silent gap between motifs & bout ldf \\
\hline \multicolumn{3}{|l|}{ Song motif features } \\
\hline Duration & $\begin{array}{l}\text { Total duration of song motif, including silent gaps } \\
\text { between syllables }\end{array}$ & \\
\hline Syllable number & Number of syllables per song motif & motif ldf \\
\hline Mean syllable duration & Mean duration of syllables within a motif & \\
\hline Mean syllable rate & Mean number of syllables per second & \\
\hline Syllable distance & Mean duration of silent gap between syllables & motif ldf \\
\hline \multicolumn{3}{|l|}{ Song syllable features } \\
\hline Syllable duration & Total duration of syllable & syllable ldf \\
\hline Syllable distance & Duration of silent gap following syllable & syllable ldf \\
\hline
\end{tabular}

The subset of features retained for inclusion in the linear discriminant function analyses are indicated.

song trait in the analysis, because the three types of songs (heterospecific, isolate, and wild-type conspecific) show differences in these parameters.

To obtain the song-bout and song-motif spectral and temporal measurements, we manually identified the start and end of every bout and motif, and SAP automatically calculated the variables. To calculate the syllable spectral and temporal measurements, we used a sound file containing a single motif and SAP segmented the syllables automatically. For the automatic analyses, we used the default parameters defined by SAP.

\subsection{Statistics}

Our analysis strategy consisted of first performing a linear discriminant function analysis of the initial tutor songs used to found the three conditions. Then, we classified the songs from new generations based on these discriminant function models. We replicated this approach at three levels of song organization: song-bouts, song-motifs, and song-syllables 


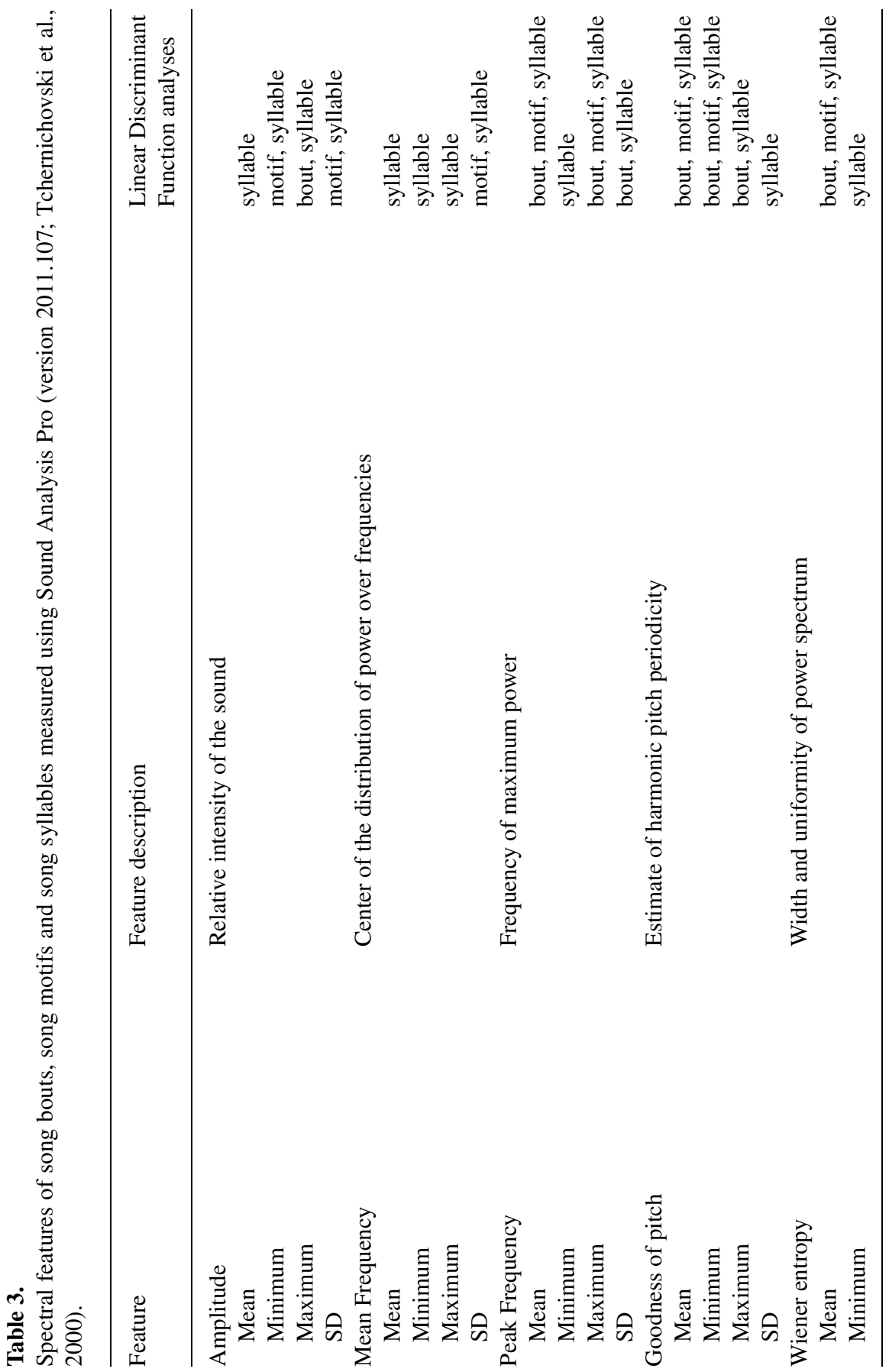




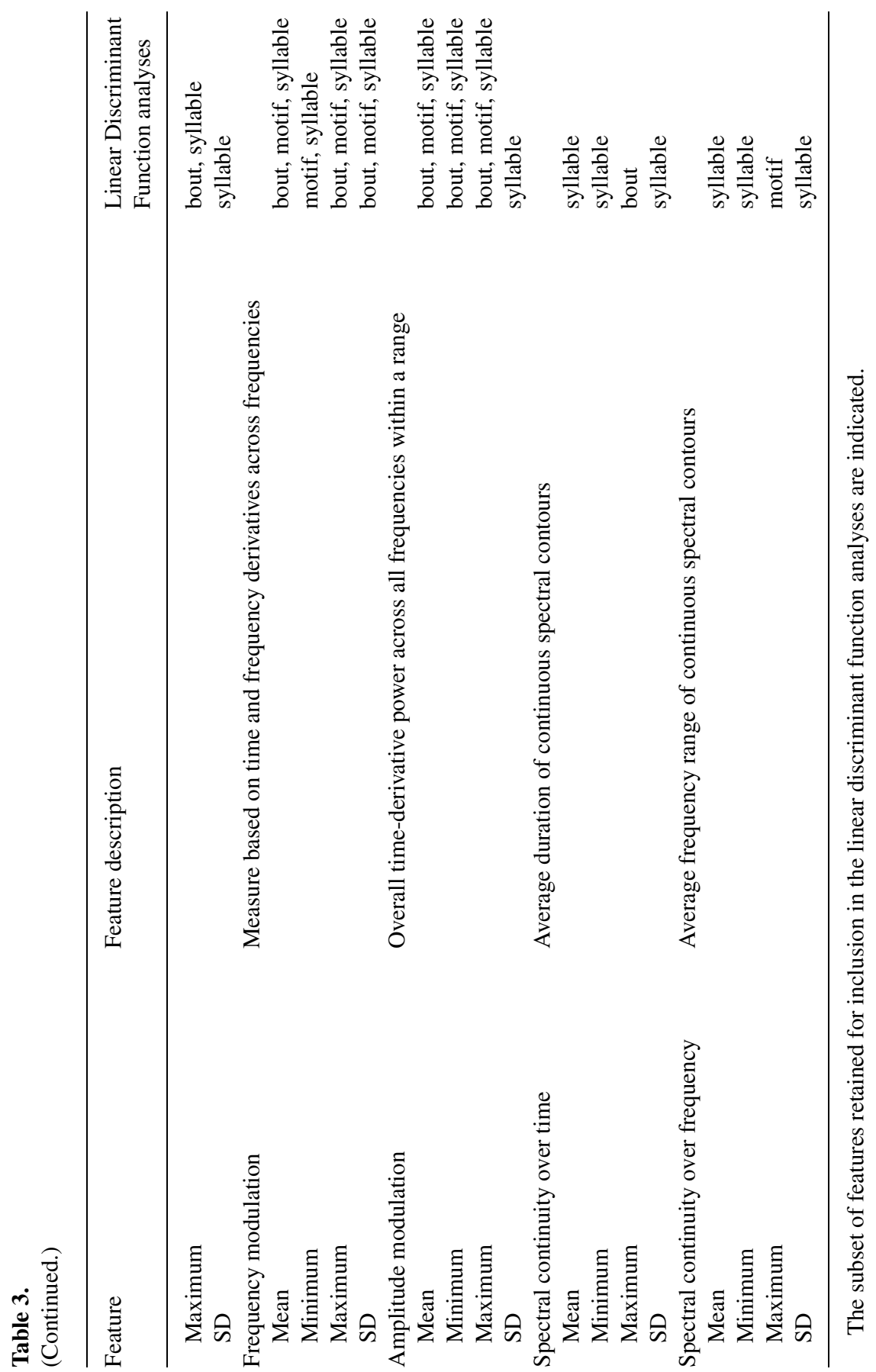


To conduct the above analyses we used Fisher's linear discriminant function (ldf) analysis implemented by SPSS 21.0 to derive three predictive ldf models based on the three levels of song analysis. Each of these three models were developed from the baseline-tutor group for each lineage (heterospecific song, isolate song, wild-type song). Then, these models were used to classify the new cases from the subsequent generations.

Before we ran the discriminant function analysis, we eliminated the variables that were redundant for each model. To do this, we developed a correlation matrix for each model that contained all the variables, then we manually removed one from each pair of variables for which the Pearson correlation value was higher than 0.50 . After the highly correlated variables were removed, we ran the discriminant functional models. A further elimination of variables was made by using the stepwise $F$ to enter $F$ to remove procedure; specifically we used the Wilks method with a $F$ of 3.84 as inclusion and $F 2.71$ for elimination of variables criterion. Therefore, only the parameters considered significant predictor variables were included in the final models. The final set of features included are indicated in Tables 2 and 3.

When the initial tutor discrimination models for the three levels of song organization were obtained, we added the subsequent generations and lineages to assign each case to one of the three tutor groups. To test that the group classification was accurate we used the leave-one-out cross-validation procedure, that sequentially removed each individual out of the calculations to derive the discriminant functions. Subsequently, that individual was classified based on these functions.

\section{Results}

The numbers of male offspring in each generation in each tutor group are indicated in Table 1. Because our sample sizes were small for the third and fourth experimental generations we provide descriptive results for these cohorts only.

Overall, we found that the lineages founded by birds singing isolate song or heterospecific song quickly evolved towards wild-type song features at all levels of song organization (song bout, song motif, syllable). However, the rates of cultural evolution varied across groups and levels of song analysis (Figure 3). 
A) Heterospecific Lineages

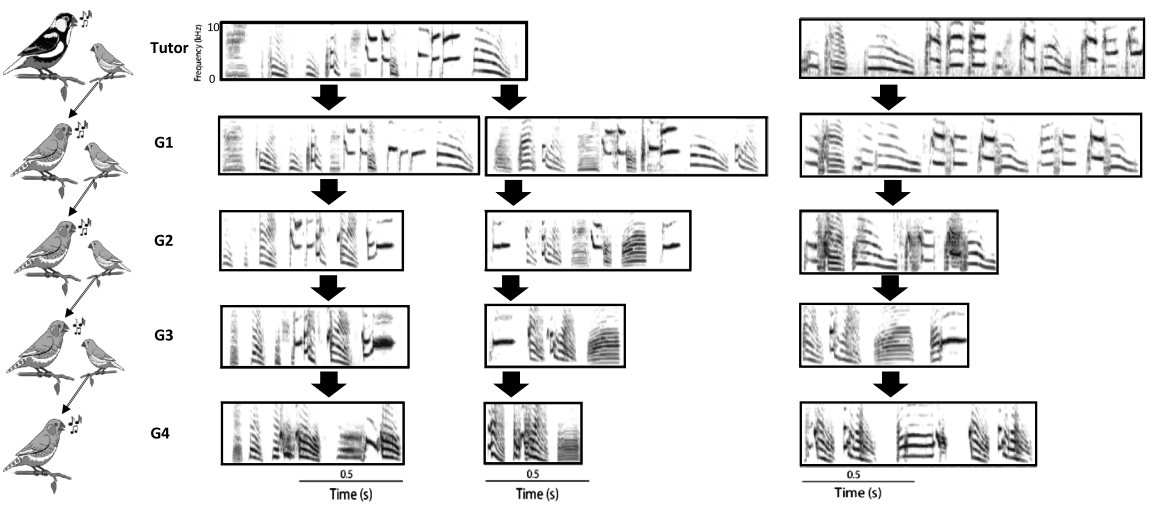

B) Isolate Lineages
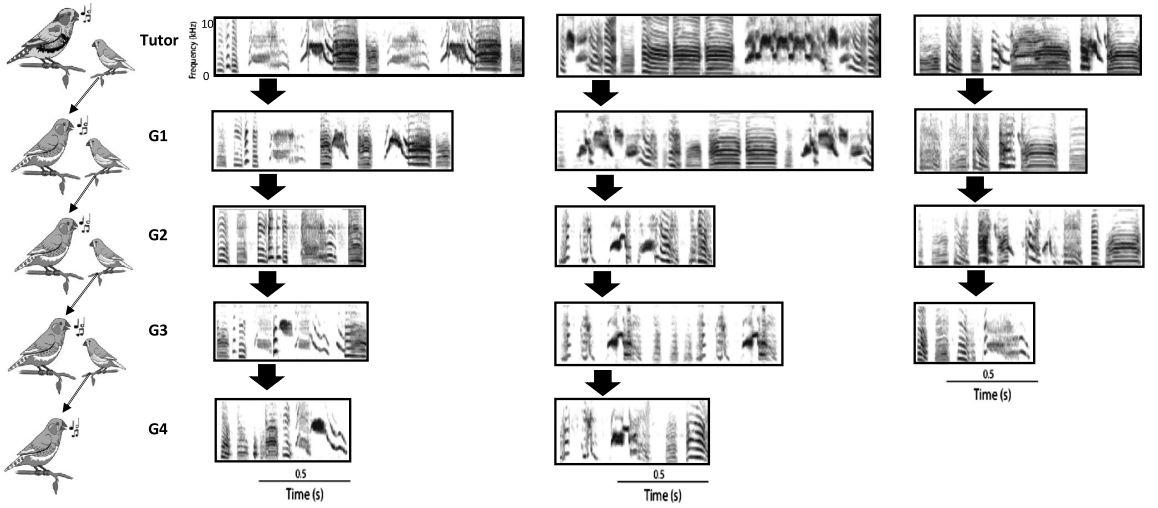

Figure 3. Example sound spectrograms from the founding tutor generation and the 4 offspring generations for the (A) heterospecific song lineages, (B) isolate song lineages and (C) wild-type conspecific song lineages. Sound spectrograms arranged vertically indicate songs from successive generations ( $\mathrm{G} 1=$ generation $1, \mathrm{G} 2=$ generation 2 , etc.). The heterospecific song examples illustrate a case of one in the tutor generation tutoring two birds in generation $1(\mathrm{G} 1)$.

\subsection{Discriminant function analysis of tutor songs:}

The grouping variable demonstrated that the tutor songs were different across tutor groups (heterospecific, isolate and wild type) and the levels of song organization (song-bout, song-motif and syllables).

\subsubsection{Coarse-grained features - song-bout analysis}

The linear discriminant function song-bout analysis model correctly classified $90.0 \%(18 / 20)$ of the tutor song-bouts. The 2 incorrectly classified 
C) Wild type Lineages

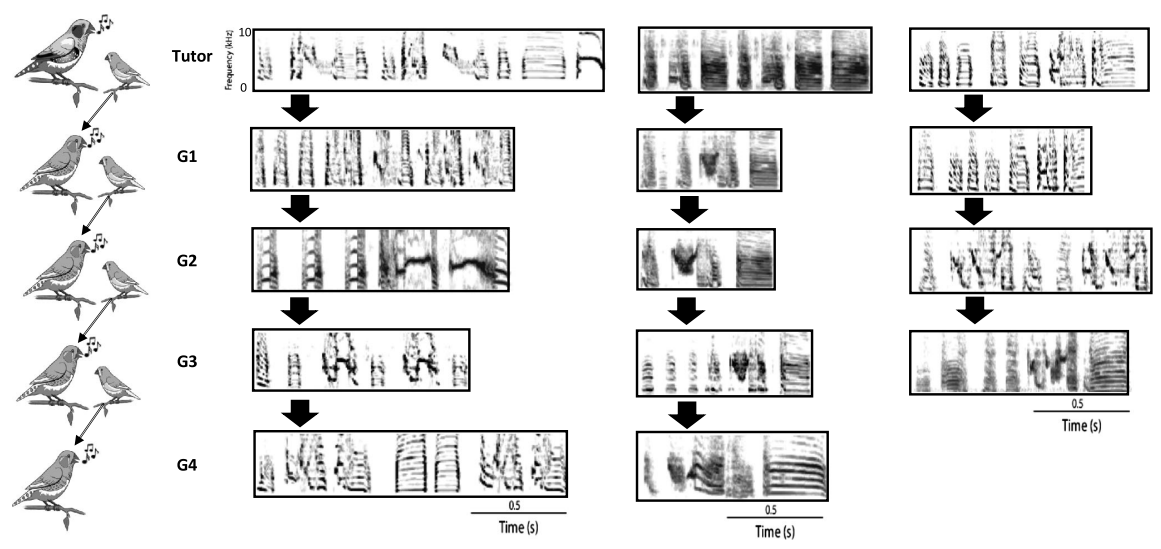

Figure 3. (Continued.)

cases included; 1 wild type song bout misclassified as isolate and 1 isolate song misclassified as wild type (Table 4). The cross-validated classification showed that overall $80 \%$ of the grouped cases were correctly classified, contrasted with $33.5 \%$ by chance. Additionally, the position of group centroids and the distribution of the individuals indicated that song bouts across the 3 tutoring lineages were distinct from each other, with isolate and wild type song-bouts more similar to each other than to heterospecific song bouts (Figure 4). The differences between the song-bouts in the tutor generation involved variables such as, song motif density per second, goodness of pitch, presence of harmonic stacks, minimum amplitude modulation, and maxima entropy (Table 5 \& 6). Generally, the isolate and zebra finch tutors sang motifs faster than the Bengalese finch tutors, and isolate and zebra finch tutor song-bouts contained a higher number of harmonic stacks than the Bengalese finch songs (Figure 3).

\subsubsection{Medium-grained features - song-motif analysis}

Linear discriminant function song motif analysis correctly classified $85.0 \%$ (17/20) of the tutor song motifs. The 3 incorrectly classified cases included, 1 wild type song-motif misclassified as an isolate song-motif and 2 isolate song misclassified as wild type (Table 4). Cross-validated classification showed that overall $78.5 \%$ of the grouped cases were correctly classified, contrasted with $33.5 \%$ by chance. Furthermore, the group centroid's position as well as the distribution of individuals indicated that song motifs across the 3 


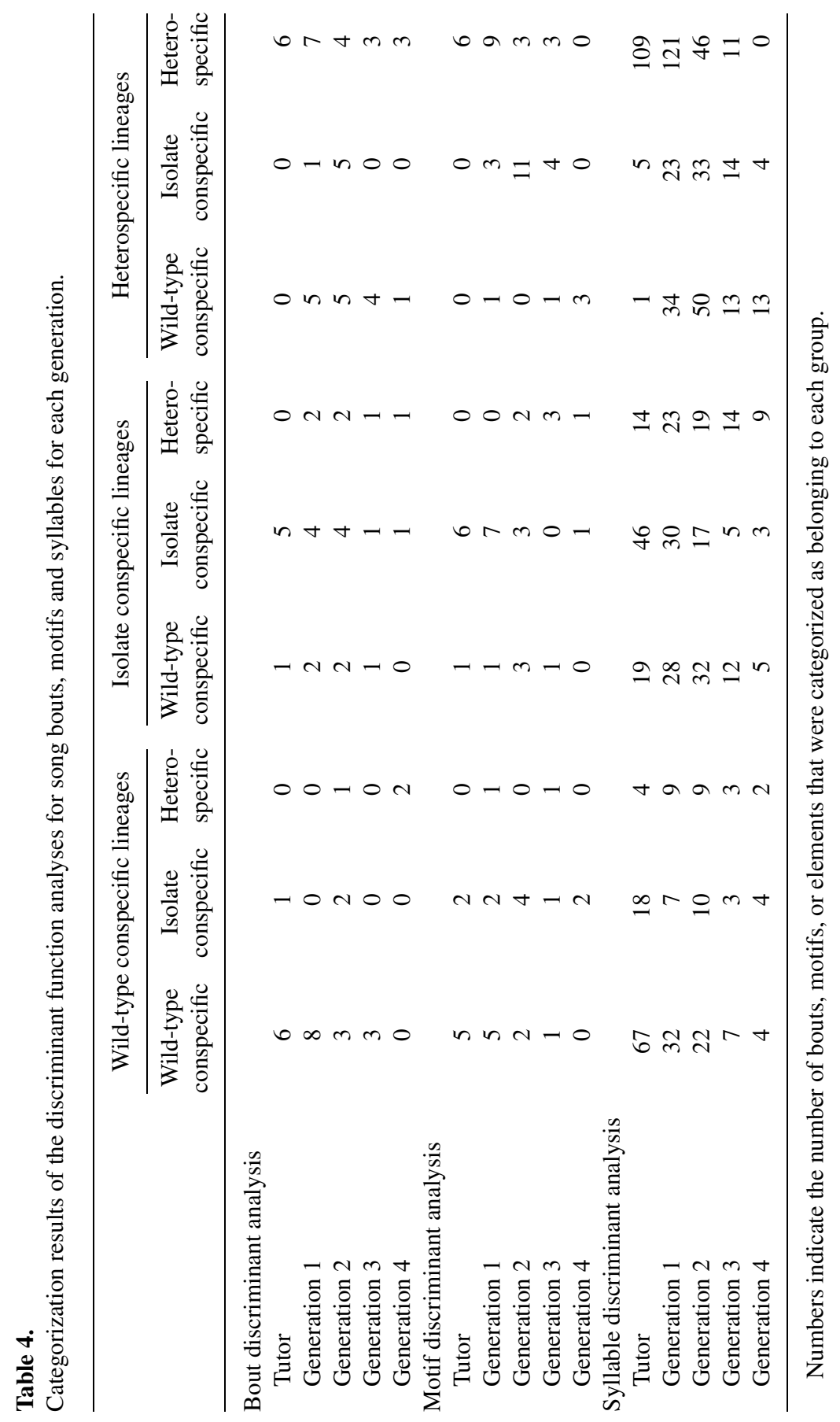


Song Bout Analysis
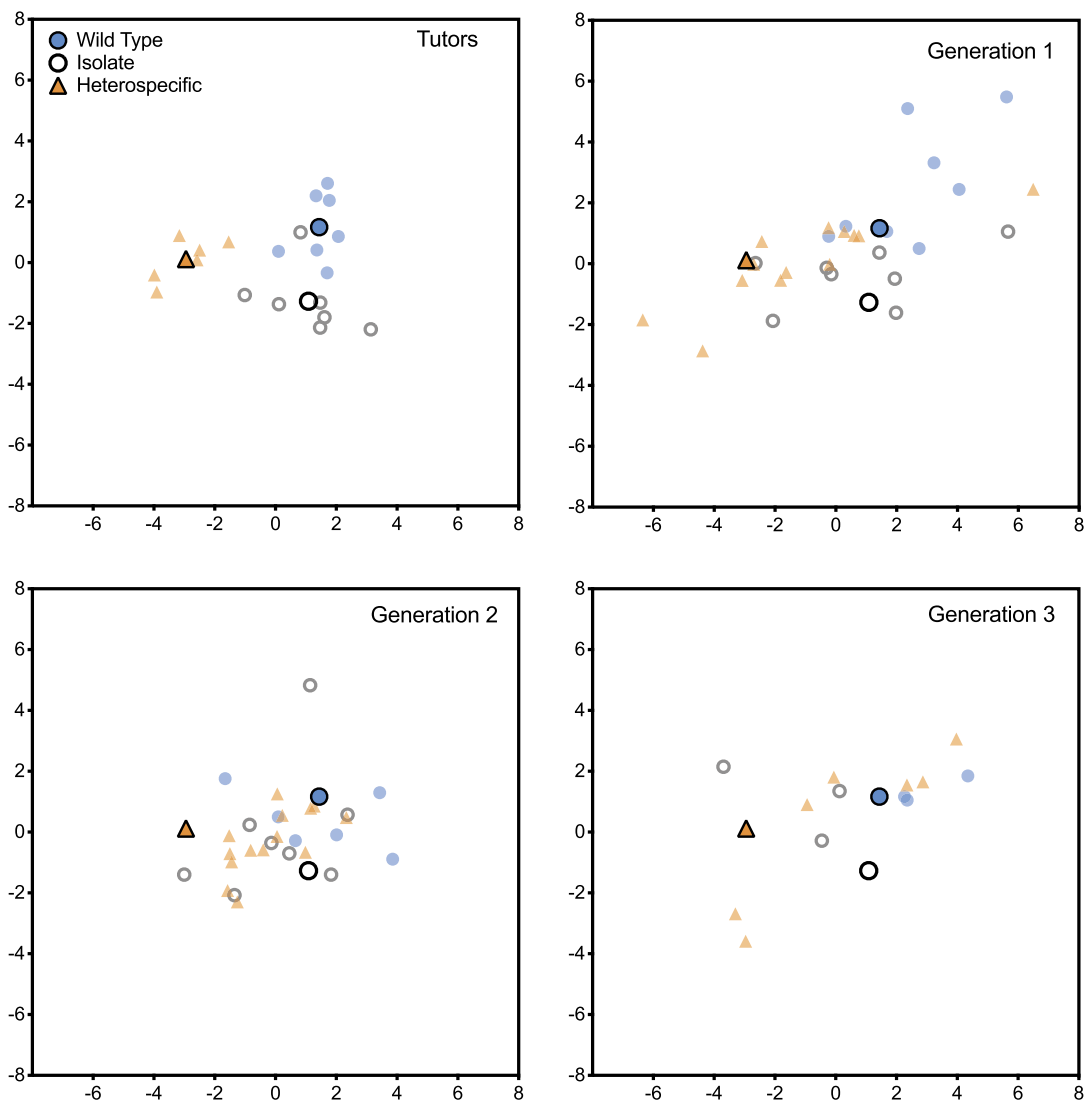

Figure 4. Plot of discriminant function values for song bouts from the tutor generation and first three generations of offspring in three tutor groups. Symbols outlined in black indicate the centroids of the tutor generation groups. Other shaded points indicate the function values for the song bouts of an individual birds. In the tutor generation the three lineages show little overlap, but over generations the function values quickly overlap.

tutoring groups were different from each other, with isolate and wild type song-motifs being more similar to each other than either isolate or wild type to heterospecific song-motifs (Figure 6). The groups' differences were based on variables such as peak frequency, frequency modulation, amplitude and mean frequency (Tables 5 and 6). Generally, zebra finch and isolate motifs had higher peak frequencies, and were louder with more power than the Bengalese finch motifs. However, the zebra finch motifs had higher frequency modulation compared to isolate or Bengalese finch motifs. 
Table 5.

Standardized canonical discriminant function coefficients per levels of song organization.

\begin{tabular}{lcr}
\hline Standardized canonical discriminant function coefficients & Function 1 & Function 2 \\
\hline Song bout & & \\
Song motif density per second & 1.072 & 0.452 \\
Goodness of pitch & 1.666 & -0.381 \\
Minima amplitude modulation & 0.991 & 0.779 \\
Maxima entropy & 0.174 & 1.257 \\
Song motif & & \\
Peak frequency & 1.22 & -0.76 \\
Frequency modulation & 0.167 & 1.157 \\
Standard deviation amplitude & 0.96 & 0.39 \\
Standard deviation mean frequency & -2.06 & -0.362 \\
Syllables & & \\
Goodness of pitch & 0.525 & 0.515 \\
Frequency modulation & 1.102 & -0.687 \\
Minima peak frequency & 0.237 & 0.383 \\
Minima spectral continuity over time & 0.226 & -0.437 \\
Maxima amplitude & 1.091 & -0.863 \\
Amplitude & -0.545 & 1.253 \\
Minima frequency modulation & -0.446 & 0.474 \\
Maxima goodness of pitch & 0.286 & -0.663 \\
Standard deviation mean frequency & -0.698 & 0.164 \\
Standard deviation amplitude modulation & -0.83 & 0.511 \\
\hline
\end{tabular}

Table 6.

Linear discriminant function analysis values per levels of song organization.

\begin{tabular}{|c|c|c|c|c|c|c|}
\hline & \multicolumn{2}{|c|}{ Song bout } & \multicolumn{2}{|c|}{ Motif } & \multicolumn{2}{|c|}{ Syllable } \\
\hline & Function 1 & Function 2 & Function 1 & Function 2 & Function 1 & Function 2 \\
\hline Eigenvalue & $4.395 \mathrm{a}$ & $1.226 \mathrm{a}$ & $6.088 \mathrm{a}$ & $0.873 \mathrm{a}$ & $1.403 \mathrm{a}$ & $0.250 \mathrm{a}$ \\
\hline$\%$ of variance & 78.2 & 21.8 & 87.5 & 12.5 & 84.9 & 15.1 \\
\hline $\begin{array}{l}\text { Canonical } \\
\text { Correlation }\end{array}$ & 0.903 & 0.742 & 0.927 & 0.683 & 0.764 & 0.447 \\
\hline Wilks' Lambda & 0.083 & 0.449 & 0.075 & 0.534 & 0.333 & 0.8 \\
\hline Chi-square & 38.529 & 12.405 & 40.085 & 9.729 & 268.844 & 54.503 \\
\hline df & 8 & 3 & 8 & 3 & 20 & 9 \\
\hline$p$ & 0 & 0.006 & 0 & 0.021 & 0 & 0 \\
\hline
\end{tabular}




\subsubsection{Fine-grained features - syllables analysis}

Finally, linear discriminant function syllables analysis correctly classified $78.5 \%$ (212/270) of the syllables. The 58 incorrectly classified cases included 19 wild type syllables misclassified as isolate syllables, 1 wild type misclassified as heterospecific. 16 isolate syllables misclassified as wild type, 5 isolate syllables misclassified as heterospecific, and 14 heterospecific syllables misclassified as isolate and 3 heterospecific misclassified as wild type (Table 4). Reclassification of cases was based on the original classification of canonical variables that was successful: $75.6 \%$ of the cases were correctly classified into their original categories, contrasted with $33.9 \%$ by chance. Furthermore, the group centroids position as well as the distribution of the cases indicated that syllables across the 3 tutoring lineages were mostly different from each other, with some overlap between the wild type and isolate syllables and wild type and isolate being more dissimilar to the heterospecific syllables (Figure 8). Generally, differences between tutor group syllables involved variables such as frequency modulation, amplitude, amplitude modulation, mean frequency, goodness of pitch, minimum peak frequency, and spectral continuity over frequency (Tables 5 and 6). Zebra finch and isolate syllables contained more harmonic stacks, higher changes in frequency modulation and the syllables were louder than the ones produced by the Bengalese finches.

\subsection{Cultural evolution of song}

To characterize changes in song features over generations, we used the discriminant functions generated using the tutor songs (above) to quantify song features of birds from each generation, within each song lineage group (wildtype, isolate, heterospecific). We repeated this process at each level of song organization (bout, song motif, syllable). In general, song features at all three levels of organization quickly converged to wild-type, consistent with prior findings (Feher et al., 2009). Both the lineages founded with isolate song tutors and heterospecific song tutors rapidly evolved toward songs whose features overlapped almost completely with wild-type song features within two generations (Figure 3). However, the progression of these features differed between levels of song organization and tutor lineage.

\subsubsection{Coarse-grained features - song-bout analysis.}

In the first generation, songs of both the isolate tutor lineage and the heterospecific tutor lineage quickly shared several features with wild-type tutor 


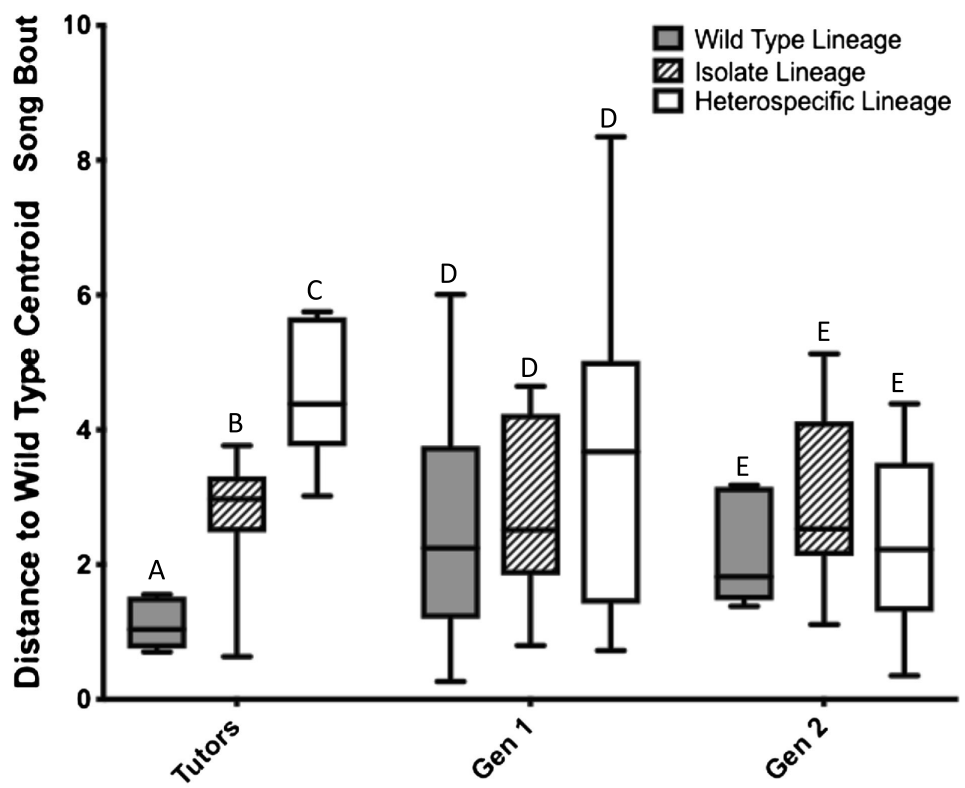

Generation

Figure 5. Distance from the centroid of the wild-type tutor group of song bouts from the tutor generation and first two offspring generations. Box plots indicate median and quartile range, and whiskers indicate range. Within each generation groups that share the same letter are not significantly different from each other. In the tutor generation the song bouts of all three groups differed from each other, but by the first generation there was no longer a significant difference across groups.

songs (Figure 4). To quantify this, we calculated the distance of each song from the centroid of the wild-type tutor songs from the tutor generation. A two-way ANOVA including the tutor generation and first two experimental generations indicated that the distance to the wild-type centroid differed between lineage groups $\left(F_{2,68}=6.4, p=0.047\right)$, and that there was a significant interaction between generation number and lineage groups $\left(F_{4,68}=2.5\right.$, $p=0.003)$. Post hoc analyses for each generation demonstrate that in the tutor generation all three groups were significantly different from each other, but that there was no significant difference between lineages in generations 1 or 2 (Figure 5).

We also tested how well our discriminant functions categorized songs to the appropriate lineage. By the first generation the functions accurately assigned all wild-type songs to the wild-type lineage, however only about half of the isolate and heterospecific lineages were correctly categorized 
Song Motif Analysis
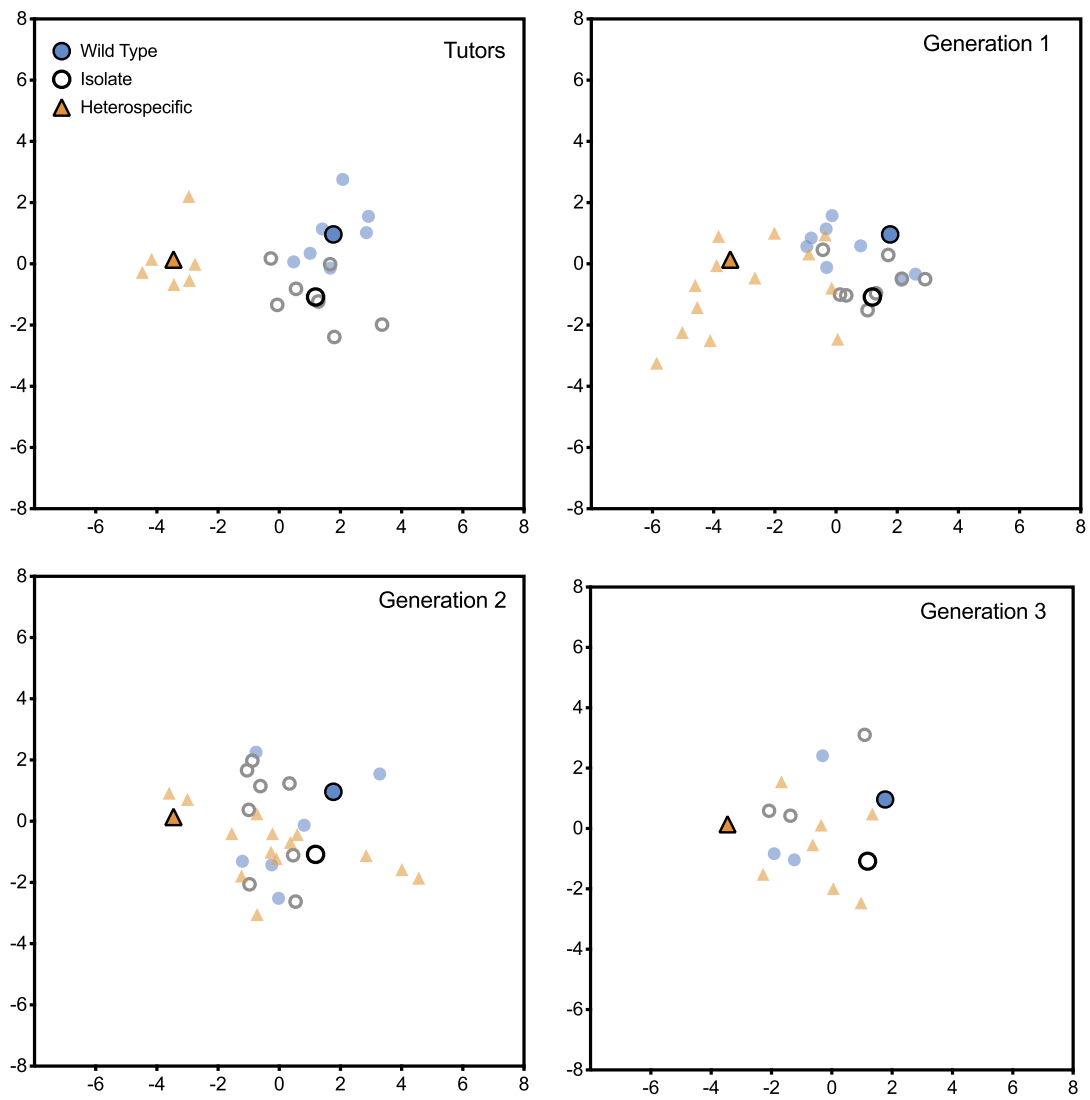

Figure 6. Plot of discriminant function values for song motifs from the tutor generation and first three generations of offspring in three tutor groups. Symbols outlined in black indicate the centroids of the tutor generation groups. Other shaded points indicate the function values for the song motifs of an individual birds. In the tutor generation the three lineages show little overlap, but over generations the function values quickly overlap. In generation 1, the isolate group song units overlap with the wild-type songs, but heterospecific songs do not overlap fully until generation 2 .

(Table 4). Combined with the above results, these data indicate that song bout features quickly evolved toward wild-type with the first generation for both isolate and heterospecific tutored lineages.

\subsubsection{Medium-grained features - song-motif analysis}

From the first generation, song-motifs of the isolate tutor lineage shared several features with wild-type tutor songs (Figure 6). In contrast, song-motifs 


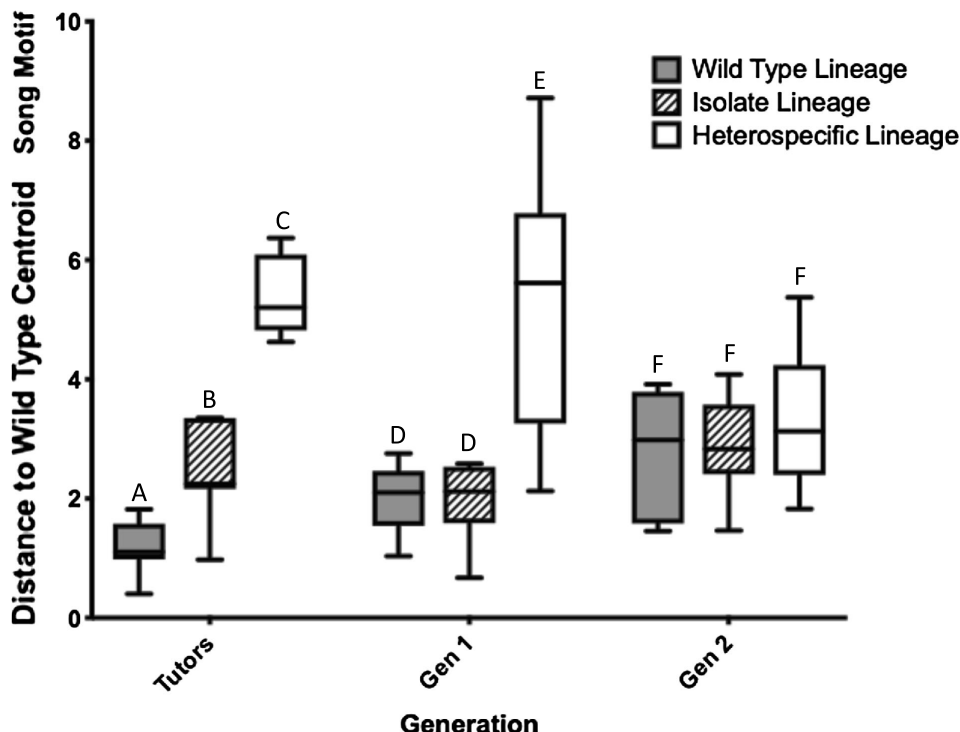

Figure 7. Distance from the centroid of the wild-type tutor group of song motifs from the tutor generation and first two offspring generations. Box plots indicate median and quartile range, and whiskers indicate range. Within each generation groups that share the same letter are not significantly different from each other. In the tutor generation the song motifs of all three groups differed from each other. In the first generation the isolate songs did not differ from wild-type, but the heterospecific songs did.

of the heterospecific tutor lineage took more than one generation to resemble features of wild type song motifs. We calculated the distance of each song from the centroid of the wild-type tutor generation songs (Figure 7). A two-way ANOVA including the tutor generation and first two experimental generations indicated that the distance to the wild-type centroid differed between lineage groups $\left(F_{2,69}=39.6, p=0.0001\right)$, and that there was a significant interaction between generation number and lineage group $\left(F_{4,69}=7.1\right.$, $p=0.0001)$. Post hoc analyses for each generation demonstrate that in the tutor generation all three groups were significantly different from each other. In generation 1 the heterospecific lineage differed from both the wild-type and isolate lineages, which did not differ from each other. There were no significant differences between lineages in generation 2 (Figure 7).

We also tested how well our discriminant functions categorized songs motifs to the appropriate lineage. The categorization of song-motifs to the wild-type category took one generation in the song-motifs produced by the isolate lineage and two generations for the song-motifs produced by the 
heterospecific lineages (Table 4). Additionally, the heterospecific lineage song-motifs shared more features with the isolate lineage than with the wildtype lineage by the second generation (Table 4). Combined with the above results, these data indicate that song motif features evolved toward wildtype but it took longer than it did for song bout features, particularly for the heterospecific lineage.

\subsubsection{Fine-grained features - syllables analysis}

At the syllable level it took until generation 2 until syllables of both the isolate tutor lineage and the heterospecific tutor lineage shared more features with the wild-type tutor lineage syllables (Figure 8). To quantify this, we calculated the distance of each song from the centroid of the wildtype tutor songs from the tutor generation (Figure 9). A two-way ANOVA including the tutor generation and first two experimental generations indicated that the distance to the wild-type centroid differed between lineage groups $\left(F_{2,813}=75.5, p=0.0001\right)$ and across generations $\left(F_{2,813}=15.5\right.$, $p=0.0001)$, and also there was a significant interaction between generation number and lineage groups $\left(F_{4,813}=10.1, p=0.0001\right)$. Post hoc analyses for each generation indicated that in the tutor generation and generation 1 all three lineages were significantly different from each other. In generation 2 there was no significant difference across wild-type and isolate lineages or between wild-type and heterospecific lineages, but there was a significant difference between isolate and heterospecific lineages (Figure 9).

We also tested how well our discriminant functions categorized syllables to the appropriate lineage. By the first generation the functions accurately assigned most of wild-type syllables to the wild-type lineage, however only $34.5 \%$ of the isolate and $19.1 \%$ of the heterospecific syllables were categorized in the wild-type lineage (Table 4). By the second generation almost half $(47.0 \%)$ of the isolate syllables were assigned to the wild-type lineage and $38.7 \%$ of the heterospecific syllables were assigned to the wild-type lineage. Collectively, with the above results, these data indicate that syllable features require at least one generation in the isolate lineage to resemble the wild-type syllables and at least two generations to resemble the wild-type syllables.

\subsection{General overview of song changes over generations}

Details of the changes over generations in temporal and spectral song features are provided in Tables 7-9. In the heterospecific song lineages, 


\section{Song Syllable Analysis}
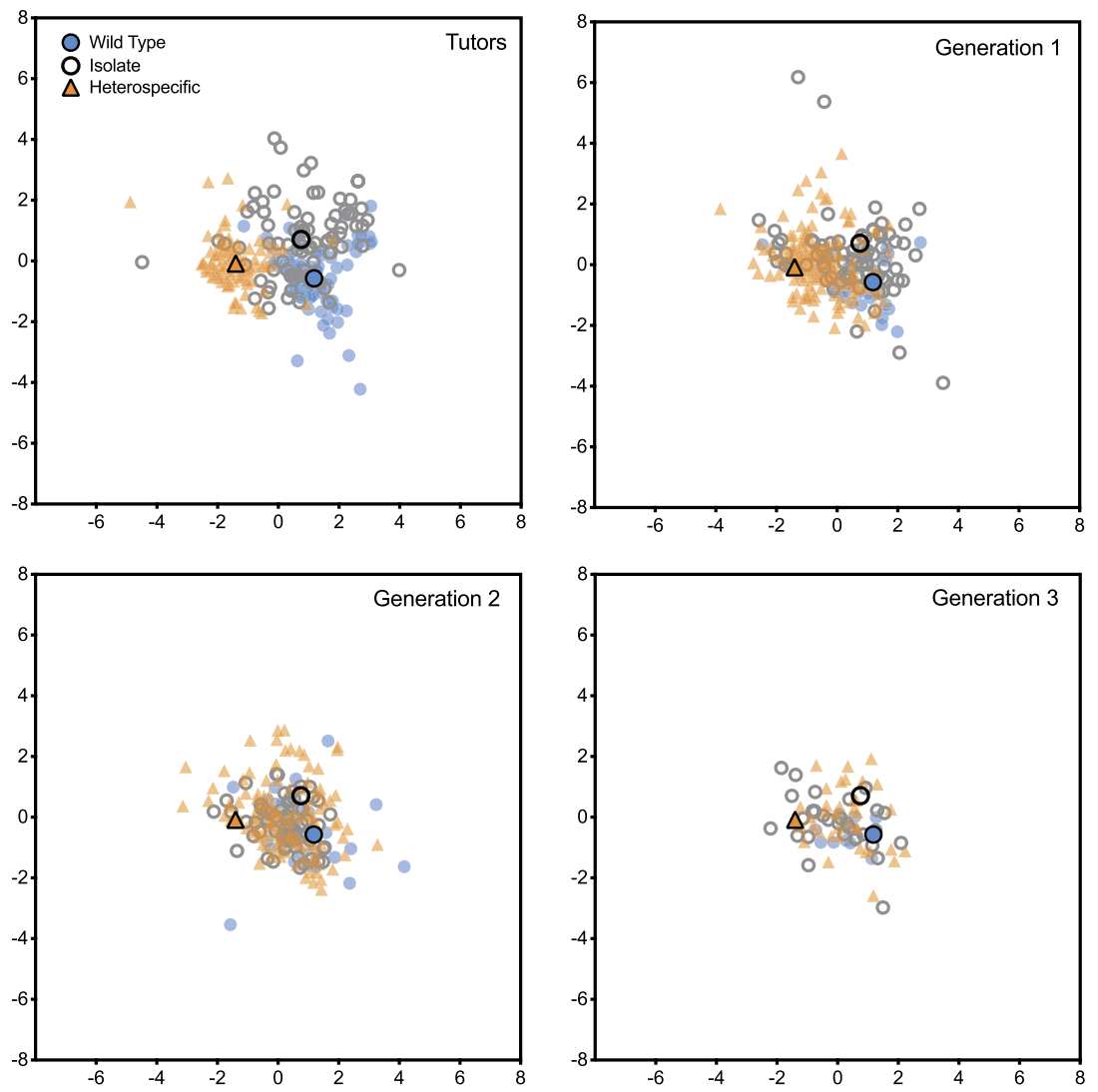

Figure 8. Plot of discriminant function values for song syllables from the tutor generation and first three generations of offspring in tutor groups. Symbols outlined in black indicate the centroids of the tutor generation groups. Other shaded points indicate the function values for the song syllables of an individual birds. In the tutor generation the three lineages show little overlap, but over generations the function values quickly overlap.

over generations song parameters drifted away from the heterospecific tutor songs. At the song-bout level, there was a reduction in the length of song-bouts, an increase in the number of song-motifs produced per second as well as an increase in the distance between song-motifs. Therefore, at the temporal level, the song-bouts produced were shorter, even though they contained the same number of song-motifs, and they were sung faster, with more distance between them. With respect to the spectral characteristics of the song-bout, the changes over generations were observed as an increased 


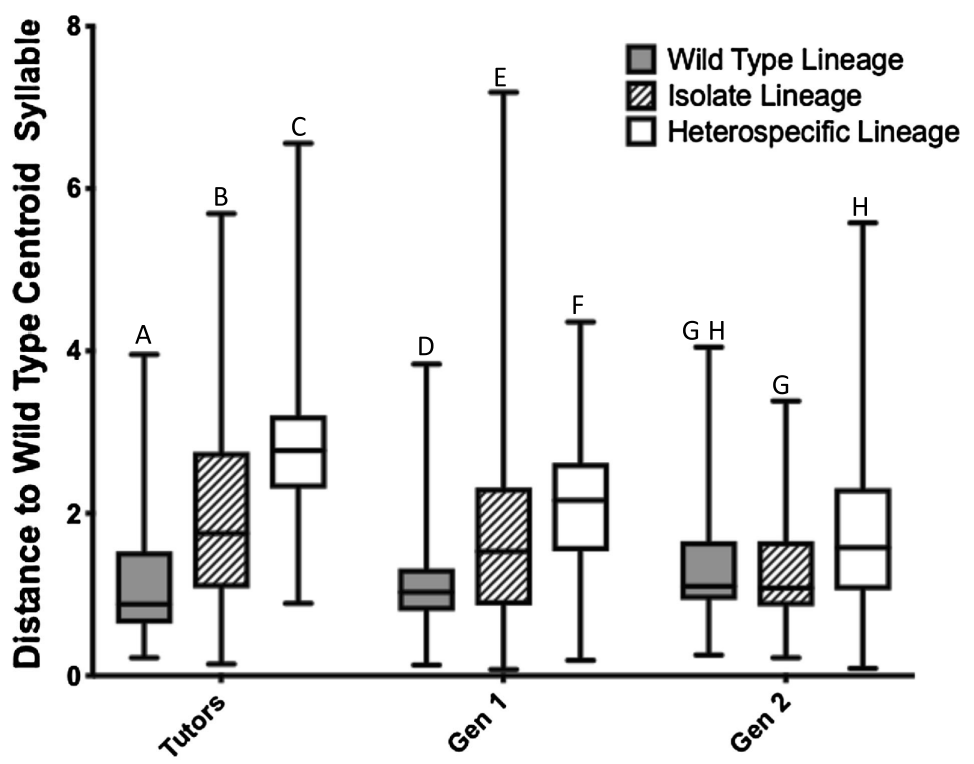

\section{Generation}

Figure 9. Distance from the centroid of the wild-type tutor group of song syllables from the tutor generation and first two offspring generations. Box plots indicate median and quartile range, and whiskers indicate range. Within each generation groups that share the same letter are not significantly different from each other. In the tutor generation the song bouts of all three groups differed from each other, but by the second generation there was no longer a significant difference across groups.

value in the goodness of pitch and entropy, and in a decreased value in the frequency modulation, resulting in higher harmonicity of the songs over generations. The changes at the level of the song-motif were characterized by shorter song bouts that contained fewer, but longer syllables, that were higher in amplitude, with increased goodness of pitch and entropy. At the syllable level, syllables over time became longer, higher in amplitude, with increased goodness of pitch, and entropy, as well as lower in FM and syllable entropy. Therefore, the syllables became louder and more harmonic.

In the isolate lineages, over generations the song parameters drifted away from the tutor isolate songs. These changes included a reduced length of the song-bout and and increase in density of the song-motifs sung per second. Thus, the songs were shorter and the song-motifs were sung faster. With respect to the spectral features, the goodness of pitch and amplitude decreased, and the frequency modulation increased so the song contained fewer har- 


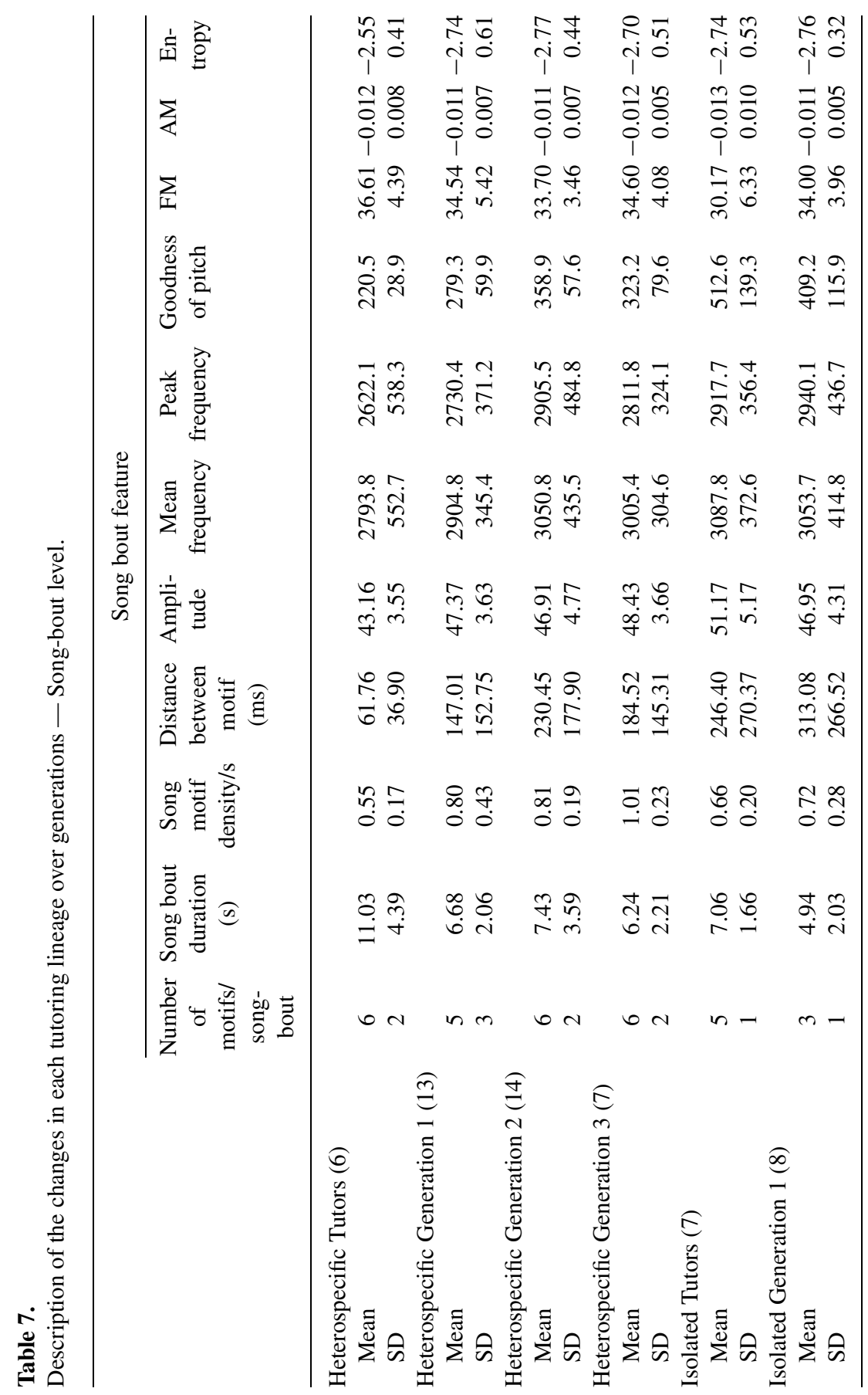




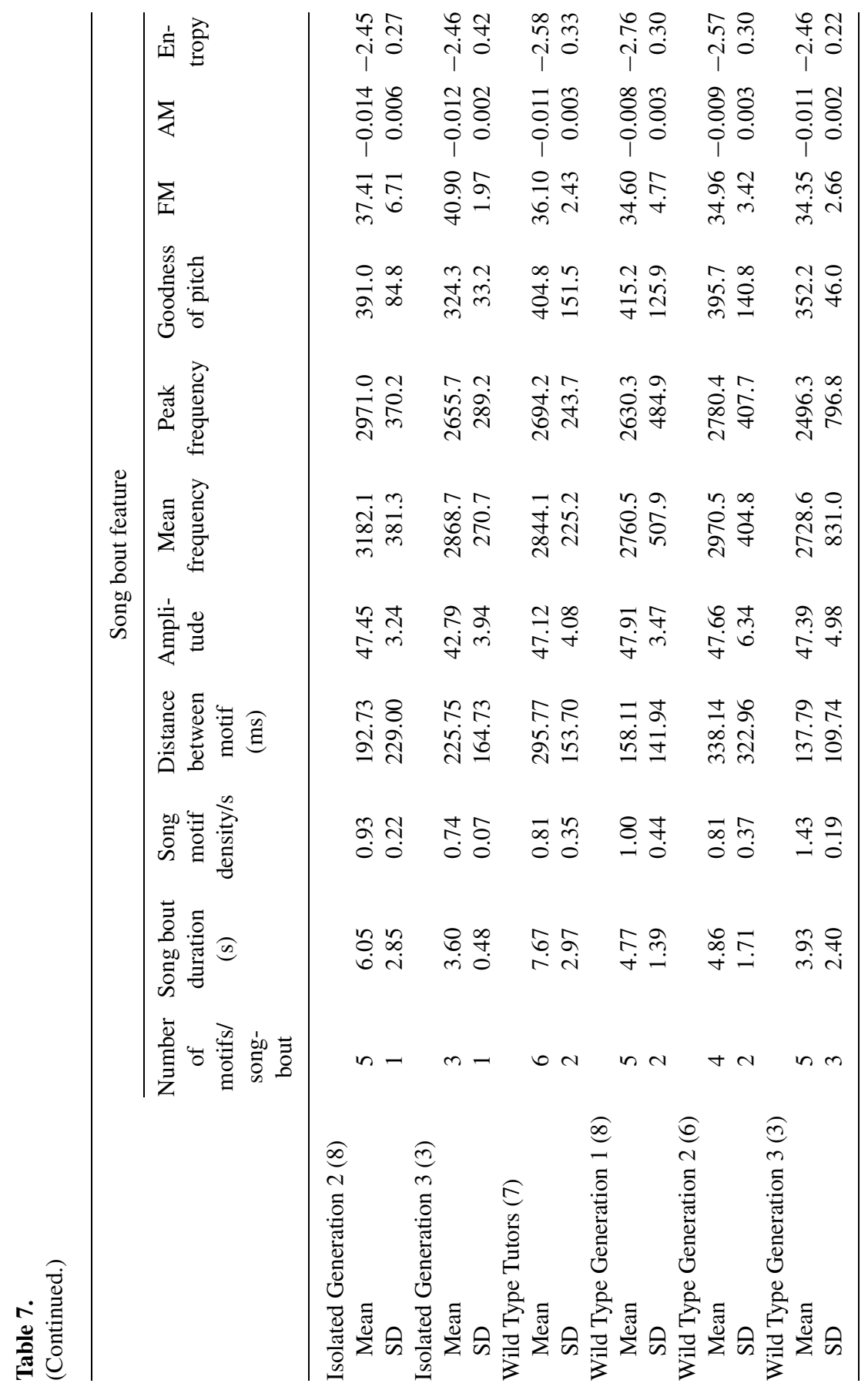




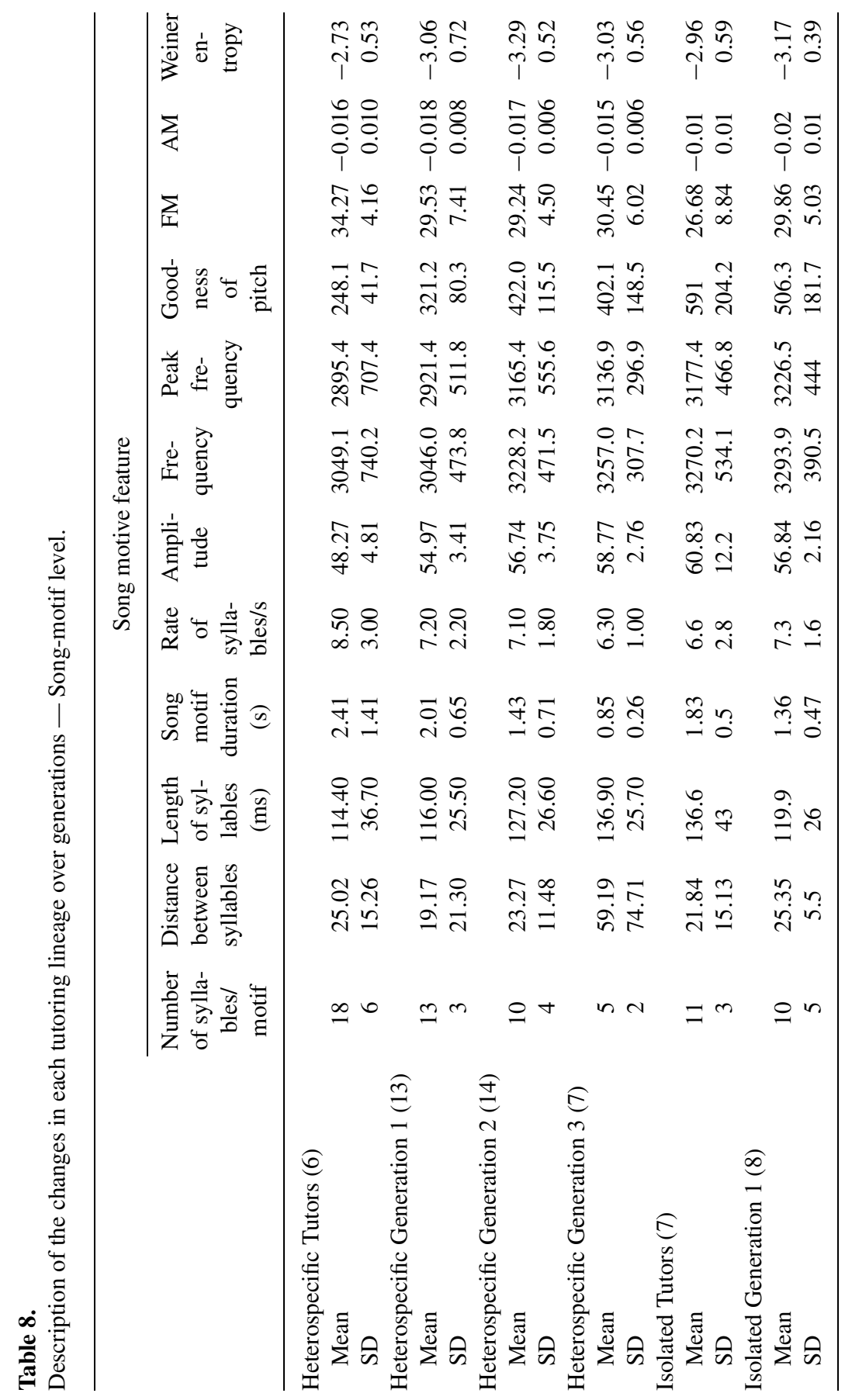




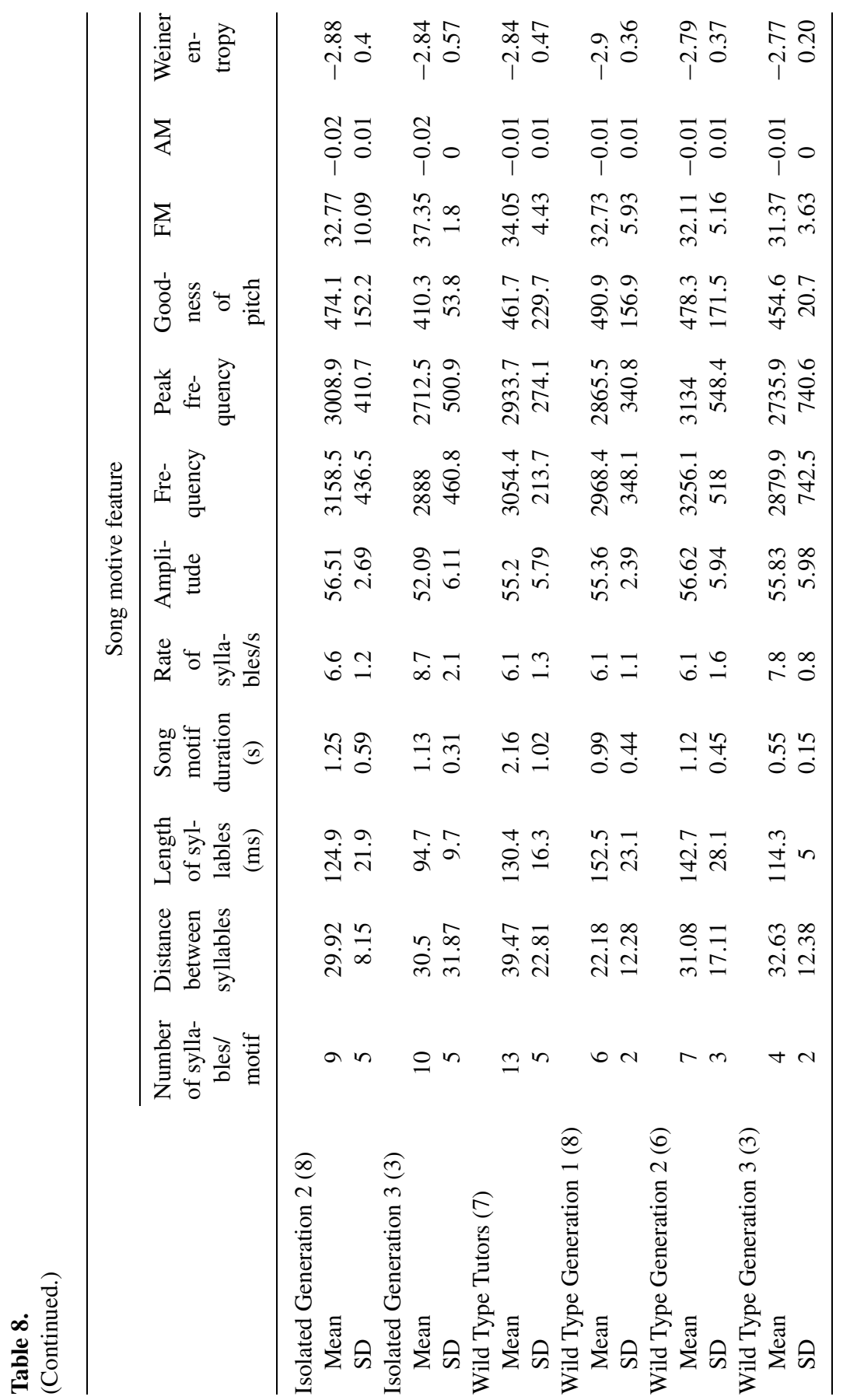




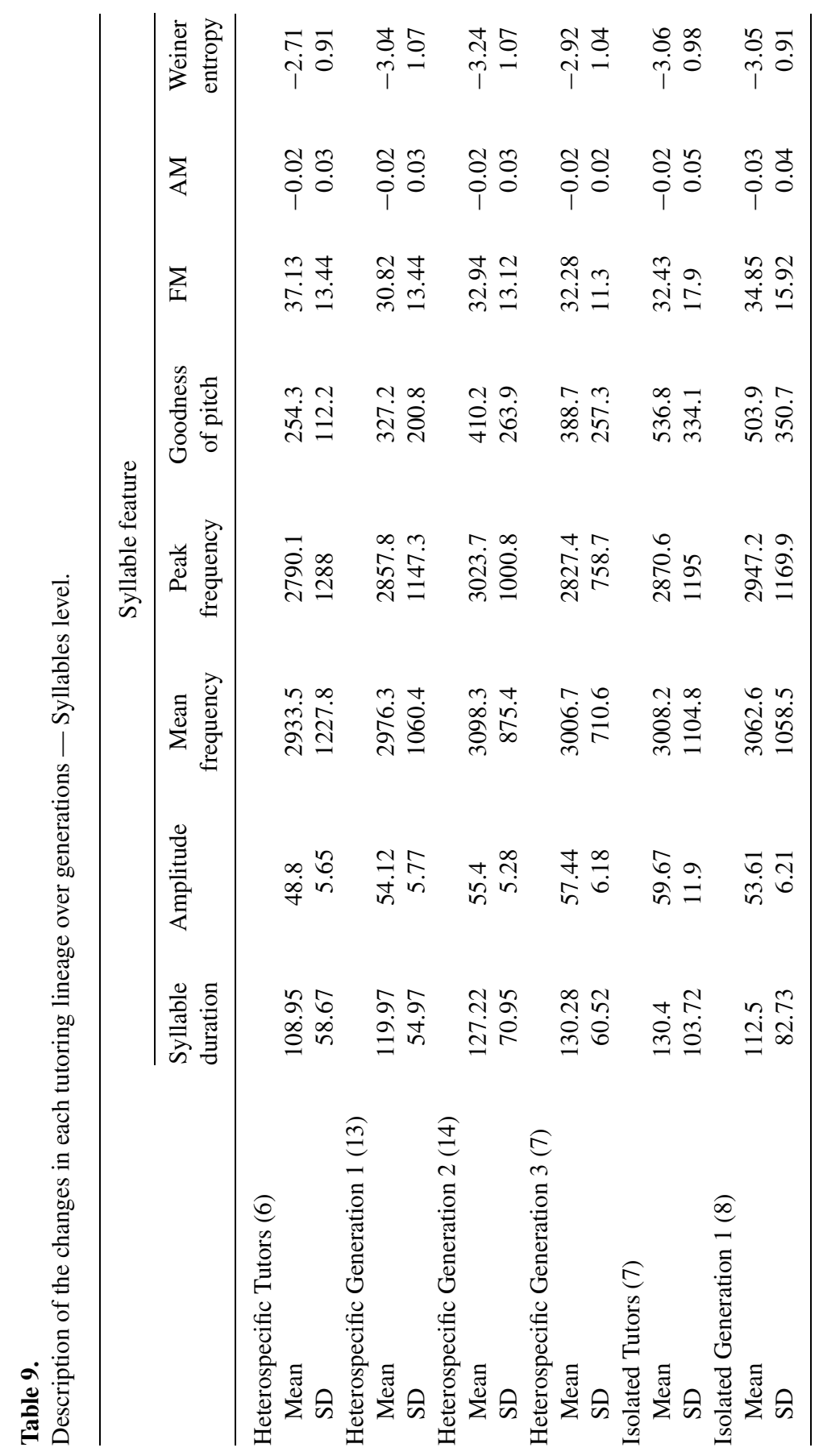




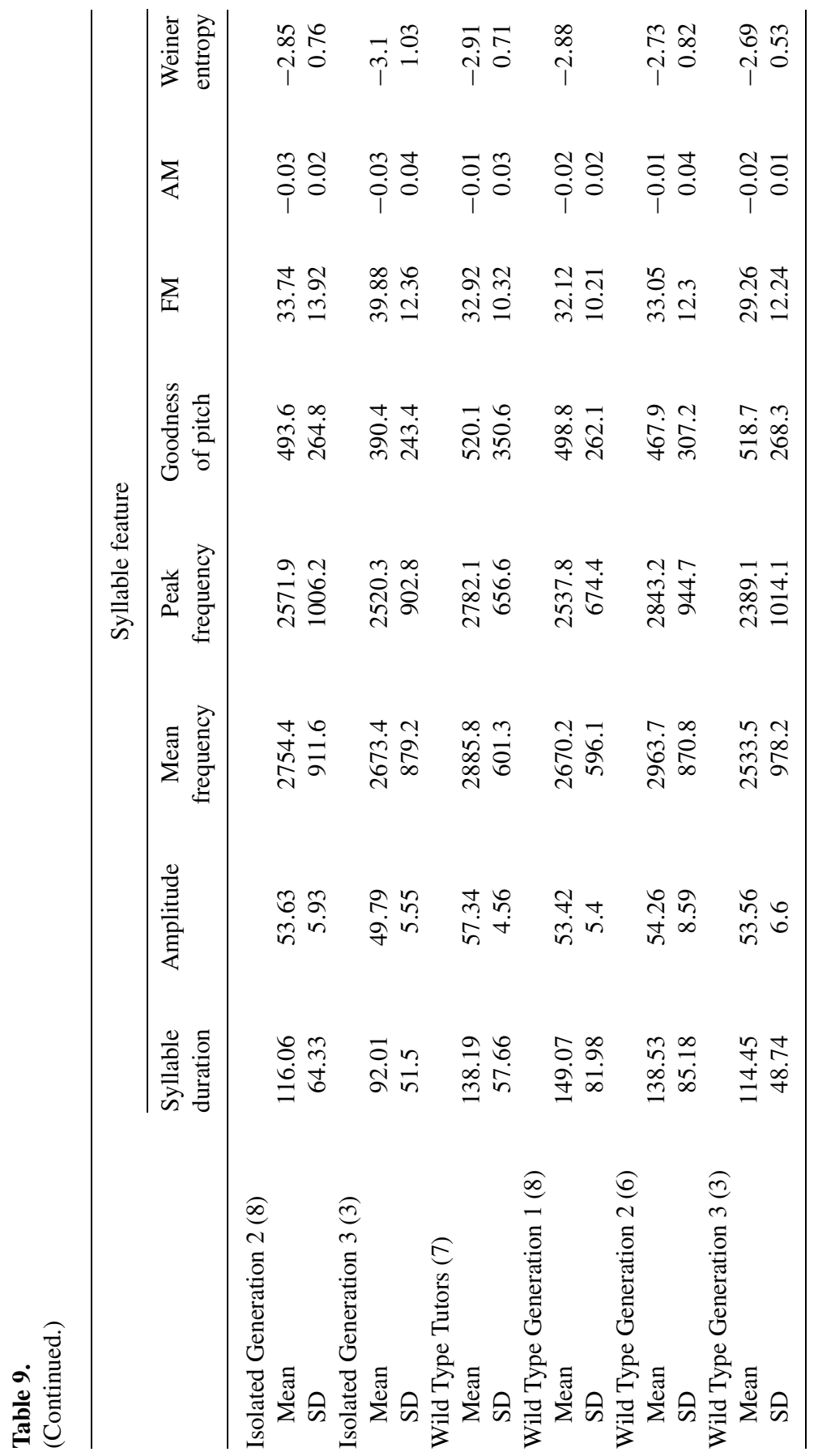


monic stacks. At the level of the song-motif, the motifs tended to decrease in length over generations, even though the number of syllables remained stable, but the syllables were shorter and the distance between them increased. Spectrally, over time, the song motifs decreased in amplitude and goodness of pitch. Therefore, it seems like the song motifs became quieter and less harmonic. At the syllabic level, the changes included shorter syllables, as well as a decrease in amplitude, and goodness of pitch. Therefore, the syllables tended to become shorter, softer and with fewer harmonic stacks.

We included a wild-type group of lineages to control for changes in song parameters that may occur during typical conspecific song learning. This group showed changes over generations, but at the group level the changes tended to centre around the tutor generation centroids, resulting in increased within-group variation, and there was some drift in song features. At the song-bout level there was a decrease in the duration of the song-bout, possibly related to a lower number of song-motifs produced, and a decrease in goodness of pitch. Therefore, the song-bouts became shorter and with fewer harmonic stacks. Song-motifs became shorter in duration and contained fewer and longer syllables. Spectrally, the song motifs became lower in frequency modulation and goodness of pitch. Finally, the syllables became lower in amplitude, goodness of pitch, and entropy. Over generations the wild-type songs tended to simplify in number of syllables and contained fewer harmonic stacks.

\section{Discussion}

Through this experimental study of the cultural evolution of song we explored the interaction between experience-independent biases in song learning and the nature of the tutor song within different lineages of zebra finches. We hypothesized that cultural evolution towards species-typical song features should be faster if the song of the founding generation had more species-typical features. In order to investigate this hypothesis, we developed three groups of lineages founded by song tutors singing wild-type zebra finch song (control group), isolate song, or song learned from a heterospecific (Bengalese finch).

We expected that, over generations, the isolate-tutored song lineages should shift toward wild-type song features faster than the heterospecifictutored lineages, as the heterospecific song contains fewer species-typical 
song features. Our results partially met our predictions. We found that both experimental lineages, isolate-tutored and heterospecific-tutored, from the first generation shifted towards wild-type song features at the song-bout level. However, at the song-motif level the shift took one extra generation in the heterospecific-tutored lineage compared to the isolate-tutored lineage. At the syllable level, it took two generations for both groups to shift towards the wild-type zebra finch song features. Therefore, the rates of cultural evolution differ across lineages and levels of song analysis.

Regardless of the founder lineage, most temporal and spectral song features shifted quickly to wild-type within one or two generations Even if the songs, after two generations, are still distinguishable by combinations of features, most of the individual feature parameters evolved quickly to completely overlap with wild type features. This suggests that zebra finches have a strong predisposition to imitate song elements with particular parameters. Even though social interactions are important during species-typical song learning (Caruso-Peck \& Goldstein, 2019), experience-independent predispositions to produce certain types of sounds likely also contributes to songlearning in zebra finches, and other species. However, using heterospecific stimuli reveals that the rate of cultural evolution to wild type depends on which songs the lineage starts with. Some features, e.g., bout length, seem to evolve more rapidly than others.

This study was based on a study developed by Fehér et al. (2009). Even though we developed three different lineages and our levels of song analysis were different, we found similar results, with regard to the rapid cultural evolution towards a wild-type song in few generations, with most of the changes over the first generation. Also, features such as frequency modulation, amplitude modulation and goodness of pitch that differentiated the isolate lineage compared to the wild-type were also characteristic in the differentiation of our wild type founder group and heterospecific founder group.

Although the features we measured, and that were determined to discriminate the tutor generation, evolved to wild type in two generations it is likely that songs from these lineages would still be discriminable based on combinations of features. To determine this would require behavioural testing to determine how zebra finches perceive and categorize the songs from different lineages. Even though the songs evolved quickly based on linear discriminant function analysis, they still sounded different to human ears. Perhaps with more generations the songs would be totally indistinguishable from wild 
type. Or it may be that some species-typical features would never emerge. Only future research would reveal this.

\section{Acknowledgements}

This research was funded by a Discovery Grant from the Natural Sciences and Engineering Research Council of Canada, and was conducted in a facility created with funding from the Canada Foundation for Innovation. We thank Alice Cui, Michela Rebuli, Pralle Kriengwatana, Tara Farrell and Haruka Wada for assistance with this project.

\section{References}

Baptista, L.F. \& Morton, M.L. (1981). Interspecific song acquisition by a white-crowned sparrow. - Auk 98: 383-385.

Baptista, L.F. \& Petrinovich, L. (1984). Social interaction, sensitive phases and the song template hypothesis in the white-crowned sparrow. - Anim. Behav. 32: 172-181.

Baptista, L.F. \& Petrinovich, L. (1986). Song development in the white-crowned sparrow: social factors and sex differences. - Anim. Behav. 34: 1359-1371.

Beecher, M.D. (2017). Birdsong learning as a social process. - Anim. Behav. 124: 233-246.

Beecher, M.D. \& Brenowitz, E. (2005). Functional aspects of song learning in songbirds. Trends Ecol. Evol. 20: 143-149.

Bolhuis, J.J., Okanoya, K. \& Scharff, C. (2010). Twitter evolution: converging mechanisms in birdsong and human speech. — Nature Rev. Neurosci. 11: 747-759.

Brainard, M.S. \& Doupe, A.J. (2002). What songbirds teach us about learning. — Nature 417: 351-358.

Carouso-Peck, S. \& Goldstein, M.H. (2019). Female social feedback reveals non-imitative mechanisms of vocal learning in zebra finches. — Curr. Biol. 29: 631-636.

Chaiken, M.L., Gentner, T.Q. \& Hulse, S.H. (1997). Effects of social interaction on the development of starling song and the perception of these effects by conspecifics. J. Comp. Psychol. 111: 379-392.

Clayton, N. (1987). Song tutor choice in zebra finches and Bengalese finches: the relative importance of visual and vocal cues. - Behaviour 104: 281-299.

Clayton, N.S. (1989). The effects of cross-fostering on selective song learning in estrildid finches. - Behaviour 109: 163-175.

Dalziell, A.H., Welbergen, J.A., Igic, B. \& Magrath, R.D. (2014). Avian vocal mimicry: a unified conceptual framework. - Biol. Rev. 90: 643-668.

Doupe, A.J. \& Kuhl, P.K. (1999). Birdsong and human speech: common themes and mechanisms. - Annu. Rev. Neurosci. 22: 567-631.

Fehér, O., Wang, H., Saar, S., Mitra, P.P. \& Tchernichovski, O. (2009). De novo establishment of wild-type song culture in the zebra finch. — Nature 459: 564-568. 
Funabiki, Y. \& Konishi, M. (2003). Long memory in song learning by zebra finches. J. Neurosci. 23: 6928-6935.

Holveck, M.J., Vieira de Castro, A.C., Lachlan, R.F., ten Cate, C. \& Riebel, K. (2008). Accuracy of song syntax learning and singing consistency signal early condition in zebra finches. - Behav. Ecol. 19: 1267-1281.

Houx, B.B. \& ten Cate, C. (1998). Do contingencies with tutor behaviour influence song learning in zebra finches? - Behaviour 135: 599-614.

Immelmann, K. (1972). The influence of early experience upon the development of social behaviour in estrildine finches. - Proc. XV Int. Ornith. Congr. (The Hague) 1970: 316338.

James, L.S. \& Sakata, J.T. (2019). Developmental modulation and predictability of agedependent vocal plasticity in adult zebra finches. - Brain Res. 1721: 1-13.

Jarvis, E.D. (2007). Neural systems for vocal learning in birds and humans: a synopsis. J. Ornithol. 148: 35-44.

King, A.P. \& West, M.J. (1983). Epigenesis of cowbird song — a joint endeavour of males and females. - Nature 305: 704-705.

Konishi, M. (1964). Effects of deafening on song development in two species of juncos. Condor 66: 85-102.

Konishi, M. (1965). The role of auditory feedback in the control of vocalization in the whitecrowned sparrow. - Ethology 22: 770-783.

Lachlan, R.F. \& Feldman, M.W. (2003). Evolution of cultural communication systems: the coevolution of cultural signals and genes encoding learning preferences. - J. Evol. Biol. 16: 1084-1095.

Lachlan, R.F. \& Slater, P.J.B. (1999). The maintenance of vocal learning by gene-culture interaction: the cultural trap hypothesis. — Proc. Roy. Soc. Lond. B: Biol. Sci. 266: 701706.

Lachlan, R.F., Janik, V.M. \& Slater, P.J.B. (2004). The evolution of conformity-enforcing behaviour in cultural communication systems. - Anim. Behav. 68: 561-570.

Ljubičić, I., Bruno, J.H. \& Tchernichovski, O. (2016). Social influences on song learning. Curr. Opin. Behav. Sci. 7: 101-107.

MacDougall-Shackleton, S.A. \& Spencer, K.A. (2012). Developmental stress and birdsong: current evidence and future directions. - J. Ornithol. 153: 105-117.

Marler, P. \& Peters, S. (1977). Selective vocal learning in a sparrow. — Science 198: 519-521.

Peters, S., Searcy, W.A. \& Nowicki, S. (2014). Developmental stress, song-learning, and cognition. - Integr. Comp. Biol. 54: 555-567.

Poirier, C., Henry, L., Mathelier, M., Lumineau, S., Cousillas, H. \& Hausberger, M. (2004). Direct social contacts override auditory information in the song-learning process in starlings (Sturnus vulgaris). — J. Comp. Psychol. 118: 179-193.

Price, P.H. (1979). Developmental determinants of structure in zebra finch song. - J. Comp. Physiol. Psychol. 93: 260-277.

Roper, A. \& Zann, R. (2006). The onset of song learning and song tutor selection in fledgling zebra finches. - Ethology 112: 458-470. 
Smith, V.A., King, A.P. \& West, M.J. (2000). A role of her own: female cowbirds (Molothrus ater) influence the development and outcome of song learning. - Anim. Behav. 60: 599609.

Soma, M.F. (2011). Social factors in song learning: a review of estrildid finch research. Ornithol. Sci. 10: 89-100.

Spencer, K.A. \& MacDougall-Shackleton, S.A. (2011). Indicators of development as sexually selected traits: the developmental stress hypothesis in context. - Behav. Ecol. 22: 1-9.

Tchernichovski, O., Nottebohm, F., Ho, C.E., Pesaran, B. \& Mitra, P.P. (2000). A procedure for an automated measurement of song similarity. — Anim. Behav. 59: 1167-1176.

Thorpe, W.H. (1954). The process of song-learning in the chaffinch as studied by means of the sound spectrograph. - Nature 173: 465-469.

Volman, S.F. \& Khanna, H. (1995). Convergence of untutored song in group-reared zebra finches (Taeniopygia guttata). - J. Comp. Psychol. 109: 211-221.

West, M.J. \& King, A.P. (1988). Female visual displays affect the development of male song in the cowbird. - Nature 334: 244-246.

Whiten, A., Ayala, F.J., Feldman, M.W. \& Laland, K.N. (2017). The extension of biology through culture. - Proc. Natl. Acad. Sci. USA 114: 7775-7781.

Williams, H., Kilander, K. \& Sotanski, M.L. (1993). Untutored song, reproductive success and song learning. - Anim. Behav. 45: 695-705. 